\title{
Gröbner Techniques for Low-Degree Hilbert Stability
}

\author{
Ian Morrison and David Swinarski
}

\section{CONTENTS}

1. Introduction

2. Hilbert Points and State Polytopes

3. Stability and State Polytopes

4. Kempf's Theory of the Worst 1-ps

5. Complementary Approaches to Checking Stability

6. Background on Wiman Curves

7. Results and Predictions from the Log Minimal Model Program

8. Results

9. Future Steps

Acknowledgments

References
We give a method for verifying, by a symbolic calculation, the stability or semistability with respect to a linearization of fixed, possibly small, degree $m$, of the Hilbert point of a scheme $X \in \mathbb{P}(V)$ having a suitably large automorphism group. We also implement our method and apply it to analyze the stability of bicanonical models of certain curves. Our examples are very special, but they arise naturally in the log minimal model program for $\overline{\mathcal{M}}_{g}$. In some examples, this connection provides a check of our computations; in others, the computations confirm predictions about conjectural stages of the program.

\section{INTRODUCTION}

We analyze the Hilbert stability of bicanonical models of certain curves $X$ of small genus with suitably large automorphism groups with respect to linearizations of fixed small degree $m$. Our examples are very special, but they have geometrically interesting applications discussed below.

Our analysis has two main novelties. First, we give a method for deducing the stability, always with respect to $\mathrm{SL}(V)$, of the Hilbert point of a subscheme $X$ of $\mathbb{P}(V)$, from a symbolic calculation of certain state polytopes. Even the possibility of such a reduction for Hilbert points of subschemes of large codimension is new. The key hypothesis we use is that $X$, as a subscheme of $\mathbb{P}(V)$, is multiplicity-free (Definition 4.5): the multiplicity, in the natural representation of $\operatorname{Aut}(X)$ on $V$, of every irreducible representation is either 0 or 1 . In our examples, $V=H^{0}\left(X, \omega_{X}^{\otimes 2}\right)$, and we say that $X$ is bicanonically multiplicity-free (Definition 4.11). Most of our examples are certain special hyperelliptic curves $\mathcal{W}_{g}$, called Wiman curves, that are well known in the literature on curves with automorphisms [Breuer 00], and nodal curves that are joins of two or more Wiman curves.

The second novelty of our examples is that they allow us to handle fixed values of the linearization degree $m$. Indeed, the values of $m$ arising in our applications are not merely fixed but quite small, typically 6 or less. In contrast, existing approaches, such as those pioneered in
2000 AMS Subject Classification: Primary 14L24, 14H10; Secondary 14D22, 13P10

Keywords: Hilbert stability, state polytope 
[Gieseker 77, Gieseker 82, Gieseker 83], have an asymptotic character and satisfy Hilbert stability only with respect to linearizations of sufficiently large degree $m$.

The bicanonical curves $X$ and small degrees $m$ in our main examples are chosen because quotients of loci in the bicanonical Hilbert schemes $\mathbb{H}$ in question are predicted to yield new log minimal models of the moduli spaces of stable curves. For further details on this connection, see [Morrison 08, 7.5] and the references cited there. A disclaimer is in order here. Examples in which $X$ is smooth show the nonemptiness of stable loci of interest in the log minimal model program in small genus but are far from producing the desired quotients. This paper deals only with our methods for checking stability and with our examples; it discusses the construction of such quotients only in outlining our plans for further work.

In addition to providing applications of our examples, the log minimal model program makes, by indirect arguments, very specific predictions about the degrees in which the bicanonical Hilbert points of certain reducible and singular $X$ will be stable, strictly semistable, and unstable. A number of our examples involve curves of these types. For some of these, we use completed stages of the minimal model program as a check on our calculations. For others, our calculations verify the program's predictions exactly, providing further evidence for them.

Our approach combines the hypothesis of multiplicityfreeness with theorems of Kempf on worst destabilizing 1-parameter subgroups (henceforth 1-ps's) to reduce checking stability for the full group $\mathrm{SL}(V)$ to checking stability with respect to a distinguished maximal torus $T$ (Corollary 4.9). There is an easy naive algorithm for checking this symbolically, but its complexity makes it impractical except in simple cases. By adapting results of Bayer and the first author on state polytopes, we give, in Corollary 3.12, an algorithm efficient enough that we are able to handle examples arising in our intended applications.

Working with small-degree $m$ is a sword that cuts both ways. On the one hand, the $m$ we work with are well below the bounds that ensure various standard uniformity hypotheses for ideals of points of $\mathbb{H}$, even those that are deformations of smooth subschemes. A typical example is that the degree- $m$ graded pieces of the homogeneous ideals do not yield the embedding of $\mathbb{H}$ as a closed subscheme of a Grassmannian needed to linearize the PGL $(V)$-action. We address these complications by replacing $\mathbb{H}$ with a multigraded Hilbert scheme $\widehat{\mathbb{H}}$ in the sense of [Haiman and Sturmfels 04].
On the other hand, our algorithm is practical only for computing state polytopes in fairly low degrees. It involves computing all the monomial initial ideals $X$ (in the coordinates giving the special torus $T$ ) and requires a Gröbner-basis calculation for each initial ideal. In fact, as the genus of $X$ - and hence the bicanonical embedding dimension - increased, we were often unable to carry even these low-degree calculations to completion because there are simply too many such ideals. To understand such examples, we use several additional, somewhat ad hoc, tricks.

The first involves a Monte Carlo strategy that computes a random subpolytope of the state polytope by computing some random initial ideals. If $X$ is Hilbert stable and we are fortunate, this subpolytope provides a proof of stability. This approach can never prove that $X$ is unstable, but we are able to do this, when necessary, by educated guesswork. Geometry - in our examples, analogies with completed stages of the log minimal model program - often suggests what a destabilizing 1-ps $\lambda$ should be, and such a guess can be checked by a single Gröbner-basis computation. Finally, the parabola trick (Proposition 5.2) uses ideas of [Hassett et al. 10] to deduce stability of Hilbert points of smooth curves in low degrees not accessible to our calculations from their stability in even lower degrees.

Here is the plan of the rest of the paper. The details of our multigraded setup for Hilbert points is given in Section 2, and the results on state polytopes we need are extended to this setting in Section 3. Section 4 reviews Kempf's results on worst one-parameter subgroups and explains how, for multiplicity-free $X$, they reduce checking stability to calculations with state polytopes. The Monte Carlo version and the parabola trick are outlined in Section 5. Section 6 recalls facts about Wiman curves and pluricanonical equations of hyperelliptic curves needed to set up the Macaulay2 calculations for our examples, and Section 7 summarizes stability properties that are either known from or predicted by the log minimal model program for $\bar{M}_{g}$. Our examples and how they fit with the log minimal model program are reviewed in Section 8. Finally, we close by listing some ideas for future work.

\subsection{Accessing Raw and Commented Source Code}

Our calculations are carried out in Macaulay2 [Grayson and Stillman 08] using the StatePolytope package of the second author, which calls the packages gfan [Jensen 08] and polymake [Gawrilow and Joswig 00, 
Gawrilow and Joswig 07] to compute intermediate results. Some preliminary calculations are performed in MAGMA [Magma 08] or GAP [GAP Group 08]. The source code of our routines and detailed output from many calculations are posted on the second author's web page at http://www.math.uga.edu/ davids/gs/gs.html. For the convenience of the reader, we have collected annotated code snippets in a separate paper [Morrison and Swinarski 09].

\section{HILBERT POINTS AND STATE POLYTOPES}

\subsection{Parameter Schemes Adapted to Low Degrees}

Fix an $(N+1)$-dimensional vector space $V$ over an algebraically closed ground field $K$, and a set of coordinates $\left\{x_{0}, \ldots, x_{N}\right\}$ identifying $V$ with $K^{N+1}$ and the homogeneous coordinate ring of $\mathbb{P}(V)$ with $S:=K\left[x_{0}, \ldots\right.$, $\left.x_{N}\right]$. Fix also a Hilbert polynomial $P$ of degree $r$ and let $\mathbb{H}$ be the Hilbert scheme of $r$-dimensional subschemes $X \subset \mathbb{P}(V)$ with Hilbert polynomial $P$.

The goal of this section is to define state polytopes of such subschemes $X$-or of their homogeneous ideals $I \subset S$-and to recall their connection to the stability of the Hilbert point of $X$ with respect to the action of $\mathrm{SL}(V)$ induced by the natural action on $\mathbb{P}(V)$. Both of these notions depend on the choice of the degree $m$ that is used to linearize this action. To make uniform sense of either the Hilbert point or the state polytope for all $X$ having a fixed Hilbert polynomial $P$ - that is, over the whole of $\mathbb{H}$ - it is necessary to take $m$ larger than the Gotzmann number [Gotzmann 78] $m_{P}$ for $P$. An easy calculation using the formulas there gives these numbers for Hilbert polynomials of curves:

Lemma 2.1. The Gotzmann number of $P(t)=a t+b$ is $m_{P}=\left(\begin{array}{l}a \\ 2\end{array}\right)+b$.

However, the applications we have in mind to stability problems arising in the log minimal model program for $\bar{M}_{g}$ (cf. [Hassett and Hyeon 09, Hassett and Hyeon 2008]) require us to work with a fixed degree $m<m_{P}$. The main goal of this section is to outline how to transfer the standard constructions to this setting. This is most conveniently achieved using the multigraded Hilbert schemes constructed in [Haiman and Sturmfels 04]. In doing this we have treated general $r$, since doing so entails no additional complications, but for the applications cited above, we will specialize to the case $r=1$ of curves.

We begin with a definition of convenience.
Definition 2.2. An $r$-dimensional subscheme $X$ of $\mathbb{P}(V)$ with ideal sheaf $I$ is called $\ell$-nice if:

1. The natural map $V^{\vee} \rightarrow \Gamma\left(X, \mathcal{O}_{X}(1)\right)$ is an isomorphism.

2. $\mathcal{O}_{X}$ is $(\ell-1)$-regular.

3. $I_{X}$ is $\ell$-regular.

This list of properties comes from a similar list of hypotheses for certain statements in [Hassett and Hyeon 08]. We note one minor change: all of the subschemes we start with are pure $r$-dimensional (though their specializations under various 1-ps's may not be), and so we have omitted this condition in Definition 2.2.

The first hypothesis may be viewed more geometrically as saying that $X$ is embedded in $\mathbb{P}(V)$ by a complete nondegenerate linear series. The second hypothesis implies that for $m \geq \ell$, the sheaf $\mathcal{O}_{X}(m)$ has no higher cohomology, and hence that its Hilbert polynomial $P(m)$ computes $h^{0}\left(X, \mathcal{O}_{X}(m)\right)$. Likewise, the third hypothesis implies that the restriction maps $S_{m} \rightarrow H^{0}\left(X, \mathcal{O}_{X}(m)\right)$ are surjective for all $m \geq \ell$ and that $I_{X}$ is generated by elements of degree at most $\ell$.

We denote by $\mathbb{H}_{\ell}$ the $\ell$-nice locus in the Hilbert scheme $\mathbb{H}$ of subschemes of $\mathbb{P}(V)$ with Hilbert polynomial $P$. Fix an $\ell$-nice subscheme $X$. We let $\widehat{R}(m)=\operatorname{dim}_{K}\left(S_{m}\right)=$ $\left(\begin{array}{c}m+N \\ m\end{array}\right)$ and $\widehat{Q}_{\ell}(m)=\operatorname{dim}_{K}\left(I_{m}\right)$ for $m \geq \ell$ and $\widehat{Q}_{\ell}(m)=0$ for $m<\ell$. In other words, $\widehat{Q}_{\ell}$ is the Hilbert function of the ideal $\widehat{I}_{\ell}$ given by truncating $I$ in degrees below $\ell$. As usual, we can recover $I$ from any $\widehat{I}_{\ell}$ by saturating. Our hypotheses imply that $\widehat{I}_{\ell}$ is generated in degree exactly $\ell$. Finally, let $\widehat{P}_{\ell}(m)=\widehat{R}(m)-\widehat{Q}_{\ell}(m)$. This is a truncation of the Hilbert function of $X$ and equals $P(m)$ only for $m \geq m_{P}$.

We denote by $\widehat{\mathbb{H}}_{\ell}$ the multigraded Hilbert scheme of ideals in $S$ with Hilbert function $\widehat{P}_{\ell}$ and denote by $[I]$ the point of $\widehat{\mathbb{H}}_{\ell}$ determined by the ideal $I$. By [Haiman and Sturmfels 04, Corollary 1.2], $\widehat{\mathbb{H}}$ is a projective scheme representing the functor of locally free families of such ideals and hence is equipped with a universal family. Their Lemma 4.1 identifies $\widehat{\mathbb{H}}_{m_{P}}$ with the usual Hilbert scheme $\mathbb{H}$, and if $\ell<m_{P}$, then truncation up to degree $m_{P}$ gives a map $i_{\ell}: \widehat{\mathbb{H}}_{\ell} \rightarrow \mathbb{H}$.

A few cautions are in order here. First, the $\ell$-nice locus in $\widehat{\mathbb{H}}_{\ell}$ is only locally closed, and it need not even be dense - there may be entire components of $\widehat{\mathbb{H}}_{\ell}$ containing no $\ell$-nice ideals.

Second, while $i_{\ell}\left(\widehat{\mathbb{H}}_{\ell}\right)$ is closed in $\mathbb{H}$ and $i_{\ell}$ is injective on the $\ell$-nice locus, the map $i_{\ell}$ need not be an embedding. 
This pathology has its origin in the fact that the ideals parameterized by $\widehat{\mathbb{H}}_{\ell}$ need not be saturated, even in degrees above $\ell$ where they are not truncated. For example, if $\mathbb{H}$ contains a point $X^{\prime}$ whose (saturated) ideal $I^{\prime}$ satisfies $\operatorname{dim}_{K}\left(I_{\ell}^{\prime}\right)>\operatorname{dim}_{K}\left(I_{\ell}\right)$ and $\operatorname{dim}_{K}\left(I_{m}^{\prime}\right)=\operatorname{dim}_{K}\left(I_{m}\right)$ for all $m>\ell$, then every choice of a $\operatorname{dim}_{K}\left(I_{\ell}\right)$-dimensional subspace of $I_{\ell}^{\prime}$ determines an ideal $I^{\prime \prime} \in \widehat{\mathbb{H}}_{\ell}$ mapping to $X^{\prime}$. Such examples can be found, for example, with $\mathbb{H}$ the Hilbert scheme of twisted cubics (cf. Example 3.11). The upshot is that we cannot replace the ideal $I$ parameterized by a point of $\widehat{\mathbb{H}}_{\ell}$ by the subscheme $X$ it determines unless we know that the degree- $\ell$ truncation of the saturation of $I$ has Hilbert function exactly $\widehat{P}_{\ell}$, as we do, by definition, over the $\ell$-nice locus.

\subsection{The Hilbert Matrix}

Henceforth we fix values of $\ell$ and $m \geq \ell$. In our applications, we often take $\ell=2$. We begin with two remarks designed to lighten our notation. First, since $m \geq$ $\ell, \widehat{P}_{\ell}(m)$ depends only on $m$, so we can and will omit the subscript $\ell$ used above. Second, we introduce many objects depending on our choice of $m$ in this section, but when there is no risk of confusion, we will omit the $m$ to simplify notation in later sections.

Let $W_{m}=\bigwedge^{\widehat{P}(m)} S_{m}$ and let $\mathbf{G r}_{m} \subset \mathbb{P}(W)$ be the Plücker embedding of the Grassmannian $\mathbf{G r}_{m}:=$ $\mathbf{G r}(\widehat{P}(m), \widehat{R}(m))$ of $\widehat{P}(m)$-dimensional quotient spaces of $S_{m}$. There is a Plücker map $g_{m}: \widehat{\mathbb{H}}_{\ell} \rightarrow \mathbf{G r}_{m}$ sending $[I]$ to $S_{m} / I_{m}$. The map $g_{m}$ has closed image, but need not be injective: for example, $g_{2}$ has the same value on the monomial ideals 3 and 4 in Example 3.11 and on the ideals 7 and 8. If, however, $I$ is generated in degrees at most $m$-in particular, for points in the $\ell$-nice locus - $g_{m}([I])$ does determine $I$.

We want to describe homogeneous coordinates $y_{A}$ of $g_{m}([I]) \in \mathbb{P}(W)$ in a form usable in tools like Macaulay2. This is most conveniently and concretely done by working with the subspace $I_{m}$ of $S_{m}$ rather than the quotient $S_{m} / I_{m}$, and using it to define $m$-Hilbert matrices $M_{I, m}$. First let $\mathcal{B}_{m}$ be the monomial basis of $S_{m}$ with a fixed ordering. Then let $\mathcal{C}_{m}(I)=\left\{p_{i} \mid i=1, \ldots, \widehat{P}(m)\right\}$ be any ordered basis of $I_{m}$ and let $M_{I, m}$ be the $\widehat{P}(m) \times \widehat{R}(m)$ matrix whose $i j$ th entry is the coefficient of the $j$ th monomial of $B_{m}$ in the equation $p_{i}$. The Plücker coordinates $y_{A}$ of $I_{m}$ are then simply the $\widehat{Q}(m) \times \widehat{Q}(m)$ minors of $M_{I, m}$ - one for each Plücker set $A$ of $\widehat{Q}(m)$ of the monomials $\mathcal{B}_{m}$. As in the discussion in [Bayer and Morrison 88 , p. 211], if $M_{I, m}^{\prime}$ is the matrix associated to a second basis $\mathcal{C}_{m}^{\prime}(I)$ and $E$ is the associated change-of-basis matrix, then $M^{\prime}=E M$, and for all $A, y_{A}^{\prime}=\operatorname{det}(E) y_{A}$. Hence:

1. The point $g_{m}([I])$ of $\mathbb{P}(W)$ defined by the collection of $y_{A}$ is independent of the choice of $\mathcal{C}_{m}(I)$.

2. Whether an individual $y_{A}$ vanishes at $g_{m}([I])$ is likewise independent of this choice.

3. We may always make this choice so that $M_{I, m}$ is in echelon form.

Example 2.3. For a monomial ideal, we may take the basis $\mathcal{C}_{m}$ to be monomial, too, and then the Hilbert matrix is particularly simple: it will have exactly one 1 in each row and be 0 otherwise. Thus, for a given $m$, there is exactly one nonzero Plücker coordinate, given by the Plücker set $A=\mathcal{C}_{m}$.

Example 2.4. Consider the ideal $I$ of two distinct points in $\mathbb{P}^{2}$, for instance $P=(1,2,3)$ and $Q=(5,1,-4)$. Let $a, b, c$ be the coordinates on $\mathbb{P}^{2}$. Then we can view $I$ as $(c-3 a, b-2 a) *(a-5 b, c+4 b)$ and take

$$
\begin{aligned}
\mathcal{C}_{2}=\left[2 a^{2}-11 a b+5 b^{2}, 8 a b-4 b^{2}+2 a c,\right. \\
\left.\quad 3 a^{2}-15 a b-a c+5 b c, 12 a b+3 a c-4 b c-c^{2}\right] .
\end{aligned}
$$

Ordering $\mathcal{B}_{S_{2}}$ as $\left[a^{2}, a b, a c, b^{2}, b c, c^{2}\right]$, we get

$$
M_{I, 2}=\left(\begin{array}{cccccc}
2 & -11 & 0 & 5 & 0 & 0 \\
0 & 8 & 2 & -4 & -1 & 0 \\
3 & -15 & -1 & 0 & 5 & 0 \\
0 & 12 & 3 & 0 & -4 & -1
\end{array}\right) .
$$

Then the Plücker point of $M_{I, 2}$ is given by the following point, in which we have indexed the Plücker sets by the pair of monomials omitted to save space:

\begin{tabular}{|r|r|r|r|r|r|}
\hline$\widehat{12}$ & 45 & $\widehat{23}$ & 55 & $\widehat{35}$ & 108 \\
$\widehat{13}$ & -95 & $\widehat{24}$ & -18 & $\widehat{36}$ & -228 \\
$\widehat{14}$ & 99 & $\widehat{25}$ & 38 & $\widehat{45}$ & 22 \\
$\widehat{15}$ & -154 & $\widehat{26}$ & -13 & $\widehat{46}$ & 55 \\
$\widehat{16}$ & 209 & $\widehat{34}$ & -83 & $\widehat{56}$ & -132 \\
\hline
\end{tabular}

Alternatively, the Plücker coordinates can be computed in Macaulay2 [Morrison and Swinarski 09, CodeSample 2].

\section{STABILITY AND STATE POLYTOPES}

\section{1. $\quad T$-States and $T$-State Polytopes}

We next want to focus on the action of $\operatorname{SL}(V) \cong \mathrm{SL}(N+$ 1) on $W:=\bigwedge^{P(m)} \operatorname{Sym}^{m} V$. The Hilbert-Mumford criterion says that $w \in W$ is $\operatorname{SL}(V)$-stable if and only if 
$w$ is $\lambda$-stable for every $1-$ ps $\lambda: \mathbb{G}_{m} \rightarrow \mathrm{SL}(V)$. If, in terms of a basis of $V$ with respect to which $\lambda$ diagonalizes as $\operatorname{diag}\left(t^{r_{0}}, \ldots, t^{r_{N}}\right)$, the point $w$ has coordinates $\left(w_{0}, \ldots\right.$, $\left.w_{N}\right)$, we set

$$
\mu^{L}(w, \lambda):=-\min \left\{r_{i} \mid i \text { such that } w_{i}^{*} \neq 0\right\},
$$

and $w$ is $\lambda$-stable if and only if $\mu^{L}(w, \lambda)<0$.

Remark 3.1. A word about the minus sign in the definition of $\mu$. Our preferred sign convention for the index $\mu$ of a Hilbert point $w$ is that of [Gieseker 82, Baldwin and Swinarski 08, Morrison 08], in which we consider the Grassmannian as parameterizing $P(m)$-dimensional quotients of $S_{m}$, given by restriction to $H^{0}\left(X, \mathcal{O}_{X}(m)\right)$, and $w$ is stable if any $\lambda$ acts with negative weight on some nonzero coordinate of $w$.

The minus sign has been inserted to compensate for the fact that here we will be calculating weights of the action of $\lambda$ on the degree- $m$ piece of the ideal $I$ of $X$ that is of dimension $\widehat{Q}(m)$. This, of course, gives rise to a quotient of dimension $\widehat{P}(m)$, and the complement of each Plücker set $A$ of monomials gives a basis of this quotient. But when we take $m$ to be small, we can no longer identify the quotient with $H^{0}\left(X, \mathcal{O}_{X}(m)\right)$ except on the $\ell$-nice locus, and it therefore seemed easier to us simply to work with $I_{m}$. This choice has no effect on the notion of $\mathrm{SL}(V)$-stability because the possibility of replacing $\lambda$ by its inverse means that $w$ is stable if and only if we can always find nonzero coordinates of $w$ on which $\lambda$ acts with weights of opposite signs.

Remark 3.2. When we are considering stability of a Hilbert point $[X]$, we will write $\mu([X], \lambda)(m)$ for the index with respect to the degree- $m$ linearization. Replacing the line bundle $L_{m}$ on $\mathbb{H}$ or $\widehat{\mathbb{H}}$ that is being linearized by the degree $m$, as we have implicitly been doing to this point, is harmless because $m$ determines $L_{m}$ up to powers.

For fixed choices of $x$ and $\lambda$, the index is represented by a polynomial in $m$ when $m$ is sufficiently large-for example, when $m \geq \ell$ if $X$ and its specialization under $\lambda$ are both $\ell$-nice (cf. [Hassett and Hyeon 08, Sections 3.7, 3.8 ] and Section 5.2 below). In our examples, we will often be interested in the roots of this polynomial and in other aspects of the dependence of the index $\mu$ on the degree $m$, so it is convenient to use a notation that highlights this dependence.

In some examples arising out of the log minimal model program for $\bar{M}_{g}$, we will want to consider rational values of $m$. For full details on rational linearizations, see [Dolgachev and $\mathrm{Hu}$ 98] or [Thaddeus 96]. However, we can interpret what our calculations say in such cases with- out recalling the full theory here. All we will use is that the weights of points with respect to such a linearization interpolate those with respect to integral linearizations in the following sense: if $[X]$ and its $\lambda$ specialization are $\ell$-nice, then $\mu([X], \lambda)(m)$ is computed by a polynomial in $m$ for any rational $m \geq \ell$. This allows us first to determine this polynomial from values at integral $m$, and then to evaluate it to find $\mu([X], \lambda)(m)$ at the rational values of $m$ that are of interest.

The connection with Gröbner theory comes via another way of expressing the stability of $w$ with respect to the maximal torus $T$ of $\mathrm{SL}(V)$ determined by a choice of basis $B$ of $V$. Any character $\chi \in \operatorname{Hom}\left(T, G_{m}\right)$ of $T$ may be written

$$
\chi\left(\operatorname{diag}\left(d_{0}, \ldots, d_{N}\right)\right)=\prod_{i=0}^{N} d_{i}^{z_{i}},
$$

where the $z_{i}$ are integers, determined, since we are in $\mathrm{SL}(V)$, up to a common shift. Further, any representation $W$ of $T$ decomposes into a direct sum of character eigenspaces $W_{\chi}$, where $w \in W_{\chi}$ if and only if $t \cdot w=$ $\chi(t) w$ for all $t \in T$.

We define the $T$-state $\operatorname{State}_{T}(w)$ of $w$ to be the set of characters for which the eigencomponent $w_{\chi}$ of $w$ is nonzero, and we define the $T$-state polytope $\mathcal{P}_{T}(w)$ to be the convex hull of $\operatorname{State}_{T}(W)$ in $\operatorname{Hom}\left(T, \mathbb{G}_{m}\right){ }^{1}$

The group of 1-parameter subgroups of $T$ is dual to its character group: $\lambda \cdot \chi$ is the $\lambda$-weight of $\chi$-the power of $t$ determined by the homomorphism $\chi \circ \lambda: \mathbb{G}_{m} \rightarrow \mathbb{G}_{m}$. Viewing $\lambda$ as giving a linear functional on $\operatorname{Hom}\left(T, \mathbb{G}_{m}\right)$, we may rephrase our discussion of the numerical criterion as saying that $w \in W$ is stable with respect to a $1-$ ps $\lambda$ in $T$ if and only if $w$ has two nonzero eigencomponents $w_{\chi}$ whose character vectors lie on opposite sides of the hyperplane on which $\lambda$ vanishes. Thus we arrive at the following characterization of GIT stability:

Criterion 3.3. A vector $w \in W$ is $T$-stable if and only if the trivial character lies in the interior of the state polytope, and is $T$-strictly semistable if and only if the trivial character lies on the boundary of the state polytope.

To interpret Criterion 3.3 for Hilbert points, first observe that each eigenspace $\left(S_{m}\right)_{\chi}$ of $S_{m}$ is spanned by a single $B$-monomial $M$, and if we normalize the choice

\footnotetext{
${ }^{1}$ This is the state polytope of [Bayer and Morrison 88, Section $2]$ defined entirely in the fixed degree $m$, as opposed to that of [Sturmfels 96, Theorem 2.5], which is the Minkowski sum of the former for all degrees up to $m$.
} 
of the $z_{i}$ above by requiring that they sum to $m$, then we may identify the character $\chi$ and the exponent vector of $M$. The Plücker coordinates $y_{A}$ on $W$ likewise give an eigenbasis, although the eigenspaces are not necessarily 1-dimensional. If we now normalize so that the $z_{i}$ sum to $\widehat{Q}(m) m$, then we can identify the corresponding character $\chi_{A}$ with the sum of the exponent vectors of the $\widehat{Q}(m)$ monomials determined by $y_{A}$. For example, in $\bigwedge^{2} \mathrm{Sym}^{2} K[a, b, c, d]$, the wedge product $a^{2} \wedge b c$ lies in the weight space corresponding to $W_{(2,1,1,0)}$.

Monomials and Plücker coordinates also diagonalize the actions of a $1-$ ps $\lambda$ of $T$ on $S_{m}$ and $W$. The weight $w_{\lambda}(M)$ of a monomial $M$ is the sum of the weights of its coordinate factors, and the weight $w_{\lambda}\left(y_{A}\right)$ of a Plücker coordinate $y_{A}$ is the sum of the weights of the monomials in it. Moreover, these weights agree with the $\lambda$-weights of the corresponding characters.

Thus, we think of the characters as lying on the hyperplane

$$
Z_{m}:=\left\{z \in \mathbb{Z}^{N+1} \mid \sum_{i=0}^{N} z_{i}=m P(m)\right\} .
$$

This identifies the trivial character with the point in $\mathbb{Q}^{N+1}$ having all coordinates equal to $\frac{m \widehat{Q}(m)}{N+1}$. In the sequel, we will denote this point by $\mathbf{0}_{m}$ and call it the barycenter of $Z_{m}$.

To simplify two notations that we will use frequently, we write $\operatorname{State}_{T, m}(I)$ and $\mathcal{P}_{T, m}(I)$ for the $T$-state and the $T$-state polytope of $g_{m}([I])$, omitting the $T$ when no confusion is possible.

Criterion 3.4. The $m$ th Hilbert point $g_{m}([I])$ of an ideal $I$ is $T$-stable if and only if $\mathbf{0}_{m}$ lies in the interior of $\mathcal{P}_{T, m}(I)$ and is $T$-strictly semistable if and only if $\mathbf{0}_{m}$ lies on the boundary of $\mathcal{P}_{T, m}(I)$.

Note that Criteria 3.3 and 3.4 test only $T$-stability. In Corollary 4.9, we will identify conditions under which we can extend this to $\mathrm{SL}(V)$-stability.

Example 3.5. The $m$ th state of a monomial ideal $I$ is a single point, since there is only one nonzero Plücker coordinate. Unless this point equals $\mathbf{0}_{m}$, the $m$ th Hilbert point of $I$ is unstable.

Example 3.6. If $X$ is a hypersurface of degree $d$ in $\mathbb{P}^{N}$, we may take $d=m$, so that $P(m)=1$, and suppress the exterior power in $W$. Both the characters appearing in the decomposition of $W$ and its Plücker coordinates are then indexed by monomials $\prod_{i=0}^{N} x_{i}^{z_{i}}$ of degree $d$, and,

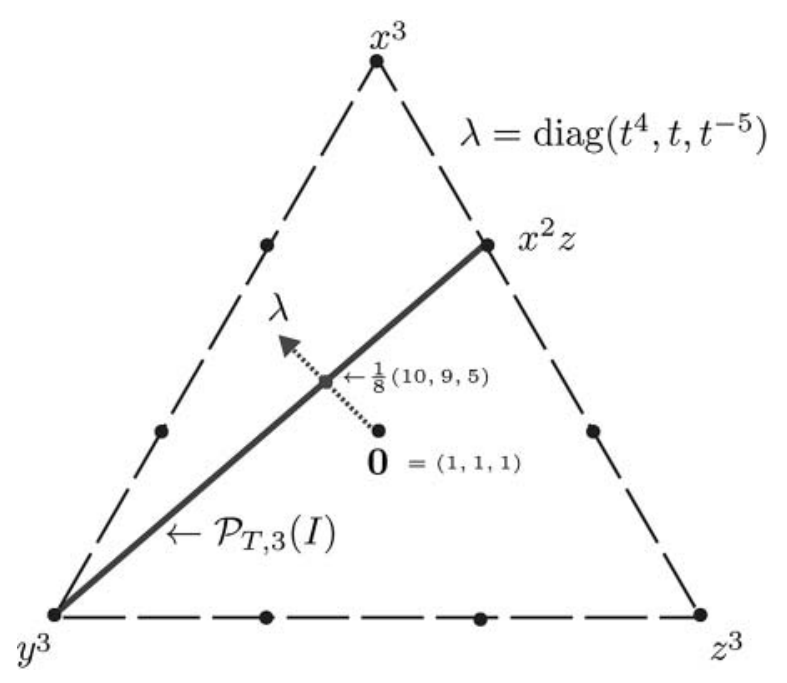

FIGURE 1. Degree-3 state polytope for the cuspidal plane cubic $\left\langle x^{2} z=y^{3}\right\rangle$.

viewed as lying in the plane $\sum_{i=0}^{N} Z_{I}=d$, form the $d$ th subdivision of an $N$-simplex.

Figure 1 shows this situation for a cuspidal plane cubic $X$ with equation $x^{2} z=y^{3}$ that is unstable with respect to the $1-$ ps $\lambda$ shown. The set of characters appearing in the decomposition is indicated by dots, and the simplex that is their convex hull is the outlined triangle. The state polytope is the line segment joining the two monomials with nonzero coefficients in the equation.

These monomials both have weight 3 with respect to the $1-$ ps $\lambda$ given by $\lambda(t)=\operatorname{diag}\left(t^{4}, t, t^{-5}\right)$, and hence this Hilbert point is unstable. The instability is reflected in the fact that $\mathcal{P}_{T, 3}([X])$ does not contain $\mathbf{0}$.

A generic hypersurface in $\mathbb{P}^{2}$ would have a twodimensional state polytope. The degeneracy of $\mathcal{P}_{T, 3}([X])$ reflects the fact that this cuspidal cubic has a $\mathbb{G}_{m}$ action (however, it is not normal). But adding an $x^{3}$ term to the equation, making the state polytope the upper subtriangle subtended by $\mathcal{P}_{T, 3}([X])$, would not affect the instability.

Example 3.7. We return to Example 2.4 and the ideal of $[1: 2: 3] \cup[5: 1:-4] \subset \mathbb{P}^{2}$. Every character with nonzero eigenspace contains a nonzero Plücker coordinate, and the state polytope is the two-dimensional hexagon pictured in Figure 2. Here the barycenter (indicated by the central solid circle) has coordinates $\left(\frac{8}{3}, \frac{8}{3}, \frac{8}{3}\right)$.

\subsection{Vertices of State Polytopes and Initial Ideals}

The number of Plücker coordinates grows quickly as the number of variables, the number of generators of the ideal, and $m$ grow. Thus it is impractical to compute the state polytope from definitions for all but the very 


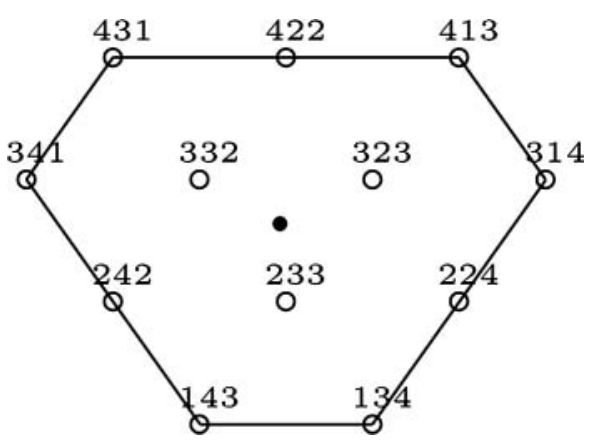

FIGURE 2. $\operatorname{State}_{2}(I)$ for two general points in $\mathbb{P}^{2}$.

simplest examples. The following results, modeled closely on analogous statements in [Bayer and Morrison 88], allow us to handle larger examples by giving a procedure for finding the vertices of $\mathcal{P}_{T, m}(I)$ that avoids the need to deal with interior Plücker coordinates.

Any $1-$ ps $\lambda$ in $T$ yields a partial order $\geq_{\lambda}$ on monomials:

$$
M \geq{ }_{\lambda} M^{\prime} \Longleftrightarrow w_{\lambda}(M) \geq w_{\lambda}\left(M^{\prime}\right) .
$$

Since the weights $w_{i}$ of $\lambda$ on $V$ are integers, there will always be ties in large degree. But in any fixed degree $m, \geq_{\lambda}$ will give a total order for all $\lambda$ not lying on a finite collection of hyperplanes. If so, we say that $\lambda$ is $m$-generic. We will say that $\lambda$ is generic if it is $m$-generic for $\ell \leq m \leq m_{P}$

Lemma 3.8. For any $I$ in $\widehat{\mathbb{H}}$ and any generic $1-p s \lambda$, there is a unique Plücker set $A_{\lambda}$ of $\widehat{Q}(m)$ monomials such that:

1. $y_{A_{\lambda}}$ is nonzero at $g_{m}([I])$.

2. If $y_{A^{\prime}}$ is any other Plücker coordinate nonzero at $g_{m}([I])$, then $w_{\lambda}\left(y_{A}\right)>w_{\lambda}\left(y_{A^{\prime}}\right)$.

Moreover, if $M_{I, m}$ is an m-Hilbert matrix for I in echelon form, then the monomials in $A_{\lambda}$ span the $>_{\lambda}$-initial ideal in ${ }_{>_{\lambda}}(I)$ of $I$ in degree $m$.

Proof: This is the content of Lemma 3.3 and Corollary 3.4.(ii) of [Bayer and Morrison 88], and the proofs given there apply verbatim in our situation.

Definition 3.9. For any generic 1-ps $\lambda$, we let $\chi_{\lambda}=\chi_{A_{\lambda}}$. In other words, $\chi_{\lambda}$ is the character given by summing the exponent vectors of the $\widehat{Q}(m)$ monomials in in $_{>_{\lambda}}(I)_{m}$. By (1) of Lemma 3.8, this character is an element of State $_{m}(I)$.

Theorem 3.10. For any $m$-generic $1-p s \lambda$, the character $\chi_{\lambda}$ is a vertex of the state polytope $\mathcal{P}_{T, m}(I)$. Conversely, if $\chi$ is any vertex of $\mathcal{P}_{T, m}(I)$, then the eigenspace $\mathrm{W}_{\chi}$ is one-dimensional and is spanned by the Plücker coordinate $y_{A_{\lambda}}$ for some m-generic $\lambda$. In particular, $\chi=\chi_{\lambda}$.

Proof: The inequality in (2) of Lemma 3.8 shows that $\sum_{i=0}^{N} w_{i} z_{i}=w_{\lambda}\left(y_{A}\right)$ is a supporting hyperplane $\left(\chi_{\lambda}\right.$ lies on it and all other $\chi^{\prime}$ in $\operatorname{State}_{m}(I)$ lie on the negative side of it) and hence proves the first claim. Conversely, any supporting hyperplane $\sum_{i=0}^{N} w_{i} z_{i}=b$ to $\chi$ may be perturbed so that the coefficients of its normal are the set of weights $w_{i}$ of a generic $1-\mathrm{ps} \lambda$. But then any Plücker coordinate $y_{A}$ lying in the $\chi$-eigenspace satisfies the conditions defining $y_{A_{\lambda}}$ in Lemma 3.8. The lemma therefore implies that there is a unique such Plücker coordinate and that $\chi=\chi_{\lambda}$. The second claim follows.

We note that in general, the dimension of $W_{\chi}$ will be quite large. Already in Figure 2, the three interior characters have 2-dimensional eigenspaces.

Theorem 3.10 is a weaker version of [Bayer and Morrison 88, Theorem 3.1], which shows that if $m \geq m_{P}$, then the set of vertices of $\mathcal{P}_{T, m}(I)$ is canonically bijective to the set of initial ideals of $I$. For the small degrees that we are treating here, where the map $g_{m}$ from an ideal to its degree- $m$ graded piece is not injective, a surjection from initial ideals to vertices is all that we can hope for-and all we need for our applications.

Example 3.11. Let $X$ be the twisted cubic in $\mathbb{P}^{3}$ with ideal $I=\left\langle a c-b^{2}, a d-b c, b d-c^{2}\right\rangle$. Then $X$ has eight initial ideals (see [Morrison and Swinarski 09, CodeSample 3]):

1. $\langle b d, a d, a c\rangle$;

2. $\left\langle c^{2}, a d, a c\right\rangle$;

3. $\left\langle c^{2}, b c, a c, a^{2} d\right\rangle$;

4. $\left\langle c^{2}, b c, b^{3}, a c\right\rangle$;

5. $\left\langle c^{2}, b c, b^{2}\right\rangle$;

6. $\left\langle b d, b^{2}, a d\right\rangle$;

7. $\left\langle b d, b c, b^{2}, a d^{2}\right\rangle$;

8. $\left\langle c^{3}, b d, b c, b^{2}\right\rangle$.

We remark that $\operatorname{State}_{2}(I)$ has six vertices: initial ideals 3 and 4 agree in degree 2, as do initial ideals 7 and 8. For any $m \geq 3$, $\operatorname{State}_{m}(I)$ has eight vertices.

By [Bayer 82, Proposition 1.8], given any multiplicative total order $>$, we can find a $1-$ ps $\lambda$ such that $>$ and $>_{\lambda}$ agree up to degree $m$. Hence we have the following corollary.

Corollary 3.12. The state polytope $\mathcal{P}_{T, m}(I)$ is the convex hull of the set of $\chi_{\mathrm{A}}$ as A runs over all Plücker sets that 
are bases for the degree-m graded piece of some initial ideal of $I$.

Conveniently, Anders Jensen's program gfan [Jensen 08] computes the set of initial ideals of $I$. Thus, if we compute the $m$ th state of each initial ideal for sufficiently large $m$, we will have the state polytope. This is what the Macaulay2 [Grayson and Stillman 08] package StatePolytope does.

We will not use the following geometric characterization of $A_{\lambda}$ but have found it helpful in thinking about the preceding results. The action of $\mathrm{SL}(V)$ on $V$ induces actions on the homogeneous polynomials of each degree on $V$ and hence on Hilbert scheme $\widehat{\mathbb{H}}$ and on the Grassmannian $\mathbf{G r}_{m}$ for which the map $g_{m}$ is equivariant. Since $\widehat{\mathbb{H}}$ is projective, we can define an ideal $J$ giving a point of $\widehat{\mathbb{H}}$ by

$$
[J]:=\lim _{t \rightarrow 0} \lambda(t) \cdot[I] .
$$

Lemma 3.8 says that $y_{A_{\lambda}}$ is the unique Plücker coordinate that is nonzero at $g_{m}([J])$ and hence that $g_{m}([J])=$ $g_{M}\left(\left[\operatorname{in}_{>_{\lambda}}(I)\right]\right)$. But all these arguments apply equally to any other degree between $\ell$ and $m_{P}$, so that in all these degrees, $J$ and $\operatorname{in}_{>_{\lambda}}(I)$ are equal. Hence $J=\operatorname{in}_{>_{\lambda}}(I)$ in degrees above $\ell$.

\section{KEMPF'S THEORY OF THE WORST 1-PS}

Let $w$ be a point of an $\operatorname{SL}(V)$ representation $W$. Already on page 64 in the first edition of [Fogarty et al. 94], Mumford conjectured that if $w$ is unstable, there is a worst destabilizing $1-$ ps $\lambda$ as measured by the index $\mu(w, \lambda)$. In this section, we review the proof of this conjecture in [Kempf 78] and [Rousseau 78], but to simplify, deal only with the linear situation we need in our applications. We follow the treatment of Kempf, which contains some complementary results that allow us to reduce the $\mathrm{SL}(V)$-stability of points $w \in W$ with suitably large stabilizer $\operatorname{Stab}(w)$ to their $T$-stability for a special torus determined by this stabilizer.

We begin by reviewing some of the background of Kempf's arguments. First, some easy covariance properties:

Lemma 4.1. For any $g \in \mathrm{SL}(V)$ :

(i) $\operatorname{State}_{T}(g \cdot w)=g \operatorname{State}_{T}(w) g^{-1}$.

(ii) $\mu(w, \lambda)=\mu\left(g \cdot w, g \cdot \lambda \cdot g^{-1}\right)$.

Proof: The first statement is [Kempf 78, Lemma 3.2(d)], and the second follows immediately from it.
Next, let $F_{\lambda}$ be the $\lambda$ weight filtration on $V$, and $P_{\lambda}$ the parabolic subgroup of block upper triangular matrices in $\mathrm{SL}(V)$ preserving $F_{\lambda}$. Equivalently, $P_{\lambda}$ consists of those $p \in \mathrm{SL}(V)$ for which the $\operatorname{limit}_{t \rightarrow 0} \lambda(t) \cdot p \cdot \lambda^{-1}(t)$ exists.

Lemma 4.2. If $g \in \mathrm{SL}(V)$ and $\mathrm{p} \in \mathrm{P}_{\lambda}$ then:

(i) $P_{g \lambda g^{-1}}=g P_{\lambda} g^{-1}$.

(ii) $p \in P_{\lambda} \Longleftrightarrow P_{p \lambda p^{-1}}=P_{\lambda}$.

(iii) If $p \in P_{\lambda}, \mu(w, \lambda)=\mu\left(w, p \lambda p^{-1}\right)=\mu\left(p^{-1} \cdot w, \lambda\right)$. Hence, $\mu(w, \lambda)=\mu(p \cdot w, \lambda)$.

Proof: The first statement follows directly from the characterization of $P_{\lambda}$ in terms of limits, and then the second follows because any parabolic subgroup is its own normalizer.

The last assertion is trickier. Our proof follows that of [Kempf 78, Lemma 3.3(e)]. The final equality follows from the first two by inverting $p$. By Lemma 4.1(ii), the second equality follows from the first. For this, the key point is the following claim: if as $t \rightarrow 0$,

$$
\lambda(t) p^{-1} \lambda^{-1}(t) \rightarrow p_{0}^{-1} \quad \text { and } \quad t^{-r} \lambda(t) \cdot w \rightarrow w_{0},
$$

then

$$
t^{-r} p \lambda(t) p^{-1} \cdot w \rightarrow p p_{0}^{-1} \cdot w_{0} .
$$

This follows because

$$
\begin{aligned}
t^{-r} p \lambda(t) p^{-1} \cdot w= & p\left(\lambda(t) p^{-1} \lambda^{-1}(t)\right)\left(t^{-r} \lambda(t) \cdot w\right) \\
& \rightarrow p p_{0}^{-1} \cdot w_{0}
\end{aligned}
$$

Since $\mu(w, \lambda)$ is the largest $r$ such that $\lim _{t \rightarrow 0} t^{-r} \lambda(t) \cdot w$ exists, the claim shows that if $p \in P_{\lambda}$, then $\mu(w, \lambda) \leq$ $\mu\left(w, p \lambda p^{-1}\right)$, and by symmetry, (iii) follows.

Replacing $\lambda(t)$ by $\lambda_{k}(t):=\lambda\left(t^{k}\right)$ for any positive integral $k$ scales all weights by $k$ without affecting their signs. Thus stability with respect to $\lambda$ is equivalent to that with respect to $\lambda_{k}$, but $\mu\left(w, \lambda_{k}\right)=k \mu(w, \lambda)$. We want to normalize the Hilbert-Mumford index $\mu$ to obtain a measure of "badness" that agrees on $\lambda$ and $\lambda_{k}$. To do this, choose a conjugation-invariant norm $\|\cdot\|$ on one-parameter subgroups - for $\mathrm{SL}(V)$, we can take $\|\lambda\|$ $:=\left(\sum_{i=0}^{N} w^{2}{ }_{i}\right)^{1 / 2}$ - and define $\widehat{\mu}(w, \lambda):=\frac{\mu(w, \lambda)}{\|\lambda\|}$. We also define $\bar{\mu}(w):=\sup _{\lambda} \widehat{\mu}(w, \lambda)$. A priori, it is not clear either that $\bar{\mu}(w)$ is finite, or if it is, that this sup is achieved. A worst $\lambda$ for $w$ is one for which the function $\widehat{\mu}(w, \lambda)$ achieves this maximum value.

In the case that $X$ is a representation $W$, [Kempf 78 , Theorem 3.4] says the following: 
Theorem 4.3. If $w$ is an unstable point of $W$, then:

1. There is an indivisible $1-p s \lambda$ such that if $\lambda^{\prime}$ is any other $1-p s$, then $\widehat{\mu}(w, \lambda) \geq \widehat{\mu}\left(w, \lambda^{\prime}\right)$. Hence $\bar{\mu}(w)$ is finite and equal to $\widehat{\mu}(w, \lambda)$.

2. The indivisible $\lambda^{\prime}$ for which $\widehat{\mu}\left(w, \lambda^{\prime}\right)=\widehat{\mu}(w, \lambda)$ are exactly those for which $\lambda^{\prime}=\mathrm{p}^{-1} \lambda_{w}$ for some $p \in$ $P_{\lambda}$. In particular, $P_{\lambda^{\prime}}=P_{\lambda}$, and we can write $P_{w}$ for $P_{\lambda}$.

3. The set of all $\lambda^{\prime}$ as in (2) is a principal homogeneous space under the unipotent radical of $P_{w}$, and every maximal torus $T$ of $P_{w}$ contains a unique such $\lambda^{\prime}$.

In view of Lemmas 4.1 (ii) and 4.2(iii), we can informally summarize this result as saying that worst oneparameter subgroups exist and are as unique as possible. The complementary result we need is this:

Proposition 4.4. [Kempf 78, Corollary 3.5] Let $w \in W$ be an unstable point with associated parabolic subgroup $P_{w}$. Then $P_{w}$ contains $\operatorname{Stab}_{w}(\mathrm{SL}(V))$.

Proof: For any $g \in \mathrm{SL}(V), g \cdot w$ is also unstable and hence determines a parabolic subgroup $P_{g \cdot w}$. The proposition will follow if we show that $g P_{w} g^{-1}=P_{g \cdot w}$, because any parabolic subgroup is its own normalizer.

By Lemma 4.1(ii) and the conjugation-invariance of the norm $\|\cdot\|$, the (indivisible) worst one-parameter subgroups for $g \cdot w$ are exactly the $g$-conjugates of those for $w$. Let $\lambda$ be one of the latter. This gives the middle equality in $P_{g \cdot w}=P_{g \lambda g^{-1}}=g P_{\lambda} g^{-1}=g P_{w} g^{-1}$, and the first and last equalities follow from Lemma 4.2(i).

Kempf applies these results to conclude stability of Chow and Hilbert points of abelian varieties and homogeneous spaces [Kempf 78, Corollaries 5.2 and 5.3]: the representations of the automorphism groups of these varieties are irreducible, so the stabilizer is not contained in any nontrivial parabolic, and hence these must be GIT stable.

There are very few examples of pluricanonically embedded smooth curves with an automorphism group acting via an irreducible representation. For instance, a full list of canonical curves with this property is found in [Breuer 00, Appendix B]; the example of highest genus is $g=14$. Examples are even rarer as $\nu$ increases. So Kempf's strategy must be modified if it is to be applied to Hilbert points of curves.

Here is how we weaken the irreducibility hypothesis.
Definition 4.5. Fix $w \in W$. We say that $w$ is multiplicityfree with respect to a finite subgroup $G$ of $\operatorname{Stab}_{\mathrm{SL}(V)}(w)$ if in the representation of $G$ on $V$, no $G$-irreducible $R$ has multiplicity greater than 1 . When, as in our applications here, $G=\operatorname{Stab}_{\mathrm{SL}(V)}(w)$, we will say simply that $w$ is multiplicity-free.

The key consequence of this property is that $V$-indeed, any $G$-invariant subspace $U$ of $V$-has a canonical decomposition as a direct sum of $G$-irreducible subrepresentations of $V$. Such a decomposition, of course, exists for any finite $G$ by complete reducibility. But when $w$ is multiplicity-free, there is, for each $R$ appearing in $V$, a canonical subrepresentation $U_{R}$ isomorphic to $R$. Every $U$ is then the direct sum of those $U_{R}$ for which $R$ occurs in $U$.

Definition 4.6. We say that a basis $B$ of $V$ or the associated torus $T=T_{B}$ determines stability for $w$ if:

1. There is a subgroup $G \subset \operatorname{Stab}_{\mathrm{SL}(V)}(w)$ such that $w$ is multiplicity-free with respect to $G$.

2. The basis $B$ is the (disjoint) union of bases $B_{R}$ for each of the $G$-irreducible representations $U_{R}$ occurring in $V$.

The justification for this terminology is the following proposition:

Proposition 4.7. If $T$ determines stability for $w$, and $w$ is $T$-semistable, then $\mathrm{w}$ is $\mathrm{SL}(V)$-semistable.

Proof: We prove that if $w$ is $\operatorname{SL}(V)$-unstable, then $w$ is $T$-unstable, by showing that then $T$ is a torus of $P_{w}$.

So suppose that $w$ is multiplicity-free and unstable. Then Proposition 4.4 says that $G$ lies in $P_{w}$ and hence fixes the associated filtration $F$. We can thus write $F$ as a strictly nested sequence $V=U_{0} \supset U_{1} \supset \cdots \supset U_{h} \supset\{0\}$ of $G$-invariant subspaces of $V$. Each of these is a direct sum of a subset of the $G$-irreducibles occurring in $V$. Therefore, the basis $B$ is compatible with the filtration $F$, and in turn, $T$ is a torus of $P_{w}$.

We now apply this to Hilbert points. Let $X$ be an $\ell$ nice subscheme of $\mathbb{P}(V)$ with ideal $I$ and let $\operatorname{Aut}_{V}(X) \subset$ $\operatorname{Aut}(X)$ be the subgroup consisting of elements that act linearly on $V$ fixing $X$. Suppose that $\operatorname{Aut}_{V}(X)$ is finite and the representation of $\operatorname{Aut}_{V}(X)$ on $V$ is multiplicityfree-in our applications $\operatorname{Aut}_{V}(X)=\operatorname{Aut}(X)$. For any $m$ $\geq \ell$, the group $\operatorname{Aut}_{V}(X)$ lies in the $\mathrm{SL}(V)$-stabilizer of 
$g_{m}([I])$, so the pair $\left(g_{m}([I]), \operatorname{Aut}_{V}(X)\right)$ is multiplicityfree in the sense of Definition 4.5, independently of $m$.

Definition 4.8. Under the hypotheses of the preceding paragraph, we say that $X$ is multiplicity-free and that any torus $T$ constructed as in Proposition 4.7 determines stability for $X$.

Combining the proposition with Criterion 3.4 gives the first assertion below. The second, which allows us to read off the worst 1-ps from the state polytope, follows by elementary arguments as in the proof of [Kempf 78, Lemma 2.3].

\section{Corollary 4.9.}

1. If $T$ determines stability for $X$, then the $m$ th Hilbert point $g_{m}([I])$ of $X$ is $\mathrm{SL}(V)$-stable (respectively $\mathrm{SL}(V)$-strictly semistable) if and only if the barycenter $\mathbf{0}_{m}$ lies in the interior (respectively the boundary) of the state polytope $\mathcal{P}_{T, m}(I)$.

2. Let $p$ be the proximum to $\mathbf{0}$ in $\mathcal{P}_{T}(w)$. If $w$ is $T$ unstable, so $p \neq 0$, then $p-\mathbf{0}_{m}$ spans a rational ray and any T-worst 1 -ps has weights lying on this ray.

Remark 4.10. Of course, multiplicity-free Hilbert points are extremely special. Consider, for example, smooth curves of genus $g \geq 2$. These usually have trivial automorphism group, and so a trivial stabilizer for any embedding. But even special curves can be multiplicityfree only for low-degree embeddings, as discussed further below. So our strategy can prove directly the stability only of low-degree models of special curves, such as those arising in our applications here. In practice, the complexity of the computations required blows up very rapidly - for an indication of just how rapidly, see Table 2 -making it practical to handle even such cases only for small $g$.

On the other hand, by the openness of GIT stability and the coarseness of the Zariski topology, proving that a single smoothable subscheme in any component of the Hilbert scheme is stable proves that a general smooth subscheme on that component is stable. As Gieseker's construction of $\bar{M}_{g}$, and many others modeled on it (cf. [Morrison 08]), shows, such a statement is often enough, when there is a main component containing smooth equidimensional subschemes, to allow the con- struction of a GIT quotient to be completed by indirect arguments.

For the rest of the paper we specialize to the case that $X$ is a curve, though many arguments will continue to apply more generally. To make this switch clear, we write $C$ for $X$, continuing to denote its ideal by $I$. In looking for examples in this case, the next step is therefore clear. Find special models $C \subset \mathbb{P}(V)$ of curves that are multiplicity-free and decide when their $m$ th-Hilbert points are stable, at least for small $m$, by computing $\mathcal{P}_{T, m}(I)$ for some $T$ that determines stability. A natural set of models to consider is that of pluricanonical models, since for these $\operatorname{Aut}_{V}(C)=\operatorname{Aut}(C)$.

Definition 4.11. We say that a nodal curve $C$ is $\nu$ multiplicity-free if its $\nu$-canonical model is multiplicityfree. We will mainly be interested in the case $\nu=2$ when we say that $C$ is bicanonically multiplicity-free.

In the sequel, we focus on bicanonical models for two reasons. First, they provide a source of tractable examples. Bicanonical embedding dimensions are small enough both so that multiplicity-free examples exist in all genera and so that it is practical to compute the relevant state polytopes when $g$ is sufficiently small. Second, as noted in the introduction, the conjectural next stages in the log minimal model program of [Hassett and Hyeon 09, Hassett and Hyeon 08] depend on understanding the stability of bicanonical Hilbert points of degree at or below 6 .

Finally, [Maclachlan 65, Theorem 4] shows that if a curve of genus $g$ has an abelian automorphism group, its order can be at most $4 g+4$. Hence no such curve can be $\nu$-multiplicity-free for any $\nu>2$ unless it has very small genus - all the irreducibles have dimension 1 , and the $\nu$-canonical series has dimension $(2 \nu-1)(g-1)$. Multiplicity-free curves with nonabelian automorphism groups seem likewise to be extremely rare. Since the sum of the squares of the dimensions of the irreducibles equals the order of the group, the sum of the dimensions themselves is typically much less, and this more than compensates for the largely theoretical extra headroom given by Hurwitz's bound of $84(g-1)$ for their orders. Our examples, reviewed in Section 6, use a family $\mathcal{W}_{g}$ of hyperelliptic curves, one in each genus $g$, called Wiman curves, whose automorphism groups are cyclic of order - surprisingly, in view of Maclachlan's bound$4 g+2$. 


\section{COMPLEMENTARY APPROACHES TO CHECKING STABILITY}

We are almost ready to describe our applications of the preceding results to check, by symbolic calculations, GIT stability of certain bicanonically multiplicity-free curves $C$ of small genus with respect to small- $m$ linearizations. Given such a $C$ with ideal $I$, we want to compute the state polytope $\mathcal{P}_{T, m}(I)$ with respect to a distinguished torus of Corollary 4.9 and check whether the barycenter $\mathbf{0}_{m}$ lies in its interior, boundary, or exterior. But in practice, already in some cases with $g=4$, this plan is impossible to carry out: there are too many initial ideals and we are unable to completely compute $\mathcal{P}_{T, m}(I)$. In this section, we explain some additional ideas that we use to settle such cases in our examples.

\subsection{A Monte Carlo Pseudoalgorithm}

Our first observation is that we can often check GIT stability without computing the entire state polytope. If we can compute any set $\mathcal{I}$ of initial ideals corresponding, in degree $m$, to a set of characters $\Xi_{m} \subset \operatorname{State}_{m}(I)$ such that the convex hull $\bar{\Xi}_{m}$ contains $\mathbf{0}_{m}$, then we know that $C$ is $m$-Hilbert semistable - even stable if $\mathbf{0}_{m}$ lies in the interior of $\bar{\Xi}_{m}$. We say that such an $\mathcal{I}$ checks $m$-semistability of $C$.

Pseudoalgorithm 5.1. To verify that $C$ is $m$-Hilbert semistable, begin with $\mathcal{I}=\emptyset$ :

1. Generate a pseudorandom weight vector $\lambda$.

2. Add the ideal $\operatorname{in}_{>_{\lambda}}(I)$ to $\mathcal{I}$ and let $\Xi_{m}$ be the associated set of characters in degree $m$.

3. If $\bar{\Xi}_{m}$ contains $\mathbf{0}_{m}$, stop. Otherwise, return to Step (1).

This is, of course, only a pseudoalgorithm because it will never terminate if $C$ is actually $m$-Hilbert unstable. In fact, even if $C$ is $m$-Hilbert semistable, we cannot be sure it will terminate: we may simply not have generated vectors in enough directions to produce a $\Xi$ that checks semistability. In practice, however, we have not encountered either problem. Guided by the predictions of the log minimal model program, we have been able to apply it only to testing $C$ that actually were $m$-Hilbert semistable. In all the examples we have run, Pseudoalgorithm 5.1 has produced a $\Xi$ that checks stability quickly, typically in a tiny fraction of the time required for our calculation of the full state polytope to complete - or fail to complete due to hardware and software limitations.
We have also been able to check the $m$-Hilbert instability of $C$ in all cases we expect it by exploiting a basic asymmetry of stability calculations: verifying semistability is hard, but proving instability is easy, if we can guess a destabilizing 1-ps. To check such a guess, it suffices to compute the in $_{>_{\lambda}}$ initial ideal (adding a tie-breaking procedure if necessary). The ideal $I$ is $m$-Hilbert unstable if and only if the monomial basis of this ideal in degree $m$ has negative weight: by Lemma 3.8, this weight equals $\mu\left(g_{m}(I), \lambda\right)$. Hassett, Hyeon, and Lee's Macaulay2 function MUm computes $\mu$ by this method (see [Hassett et al. 10] for both the code and documentation).

In our examples, the log minimal model program suggests what 1-ps to try. It gives a geometric description of those curves whose instability it predicts that suggests both the filtration and the weights of a candidate destabilizing 1-ps $\lambda$. These candidates have proven to be destabilizing in all our examples. In practice, we can often even check instability with respect to these $\lambda$ deductively, because $\lambda$-weights have a geometric interpretation. This pattern is familiar to those who have computational experience, symbolic or deductive, with Hilbert stability.

\subsection{The Parabola Trick}

For a fixed curve $C \in \mathbb{P}(V)$, the complexity of computing the state polytope $\mathcal{P}_{T, m}(I)$ grows quite rapidly with $m$ because $\left(\begin{array}{c}m+N \\ m\end{array}\right)$, the number of monomials of degree $m$, grows like $N^{m}$ for $N \gg m$. This often means that we can check $\ell$-Hilbert stability for some $\ell$ but not $m$-Hilbert stability for $m$ a somewhat larger, but still small, degree of greater geometric interest. Typically $\ell=2$, and with applications to the log minimal model program for $\bar{M}_{g}$ in mind, $m \leq 6$. The following proposition sometimes lets us deduce what we want to know from what we can compute.

The key technical tool is a lemma due to Hassett and Hyeon:

Proposition 5.2. (The parabola trick.) Let $\lambda$ be a nontrivial 1-ps. Suppose that $C$ and its $\lambda$ specialization are both $\ell$-nice. Then the weight function $\mu([C], \lambda)(m)$ is computed by a quadratic polynomial for $m \geq \ell$, and this polynomial has the form $a(m-1)(m-r)$ for some rational a and $r$. In particular, any two values of $\mu([C], \lambda)\left(m^{\prime}\right)$ with $m^{\prime} \geq \ell$ determine $\mu([C], \lambda)(m)$ for all $m \geq \ell$.

Proof: For $\ell=2$, this follows from [Hassett and Hyeon 08, Proposition 3.17], but the same proof works, mutatis mutandis, for any $\ell \geq 2$. 
We apply this trick to check both instability and stability. A typical example of the latter use is the following:

Corollary 5.3. Suppose $C$ is 2-nice, and that we can find a set $\mathcal{I}$ of initial ideals that are generated in degree $\ell$ and such that for all $m$ between $\ell$ and $m_{P}$, the corresponding set $\left\{\operatorname{State}_{m}\left(I_{j}\right) \mid I_{j} \in \mathcal{I}\right\}$ contains $\mathbf{0}_{m}$ in its convex hull. Then $C$ is $m$-Hilbert stable for all $m \geq \ell$.

The Gotzmann number $m_{P}$ grows very quickly with the genus of the curve, and therefore it is natural to wonder whether perhaps a smaller set of degrees $m$ might suffice in the corollary. The following example dashes any such hopes.

Example 5.4. Let $\mathcal{W}_{4}$ be the genus- 4 Wiman curve discussed in Example 8.2 below. Let $I$ be the ideal of this curve under its bicanonical embedding (see [Morrison and Swinarski 09, CodeSample 6]). The state polytope of $I$ is a subset of $\mathbb{R}^{9}$. There is a set $\left\{I_{j}\right\}_{j=1}^{9}$ of nine initial ideals of $I$ that are generated in degrees $\leq 7$. Hence $I$ and all the $I_{j}$ are 7 -nice. For $4 \leq m \leq 36$, but not for $37 \leq m$ $\leq 64$, the set $\left\{\operatorname{State}_{m}\left(I_{j}\right)\right\}_{j=1}^{9}$ contains $\mathbf{0}_{m}$ in its convex hull. Thus we cannot apply the parabola trick to conclude stability from this set of initial ideals (although, as we explain in Example 8.2 below, we can find another set of initial ideals that does establish stability).

Remark 5.5. In Sections 2 and 3, we emphasized the changes that must be made to the theory as it appears in the literature in order to accommodate low degrees $m$. But our approach also applies (in any dimension $r$ ) to degrees above the Gotzmann number $m_{P}$, and via these may be used to determine Chow stability. The idea is straightforward. Fix a saturated ideal $I$ corresponding to an $r$-dimensional subscheme $X$. Given any $1-$ ps $\lambda$, let $\xi_{m}$ be the character associated to $\mathrm{in}_{>_{\lambda}}(I)$ in degree $m$. For sufficiently large $m, \xi_{m}$ is represented by a vector of polynomials in $m$, and we may define a scaled limit $\xi_{\infty}=\lim _{m \rightarrow \infty} \frac{k !}{m^{k}} \xi_{m}$. By [Kapranov et al. 92, Theorem 3.3 , the Chow polytope (in [Kapranov et al. 92], simply the state polytope) Chow $(I)$ may then be defined either as the convex hull of these scaled limits for all $\lambda$, or as the scaled limit of the degree- $m$ state polytopes of $I$. Thus, we may compute Chow polytopes by computing state polytopes in $r+1$ sufficiently large degrees $m$, interpolating in each coordinate, and computing the scaled limit.

\section{BACKGROUND ON WIMAN CURVES}

Looking ahead to the next section, our main source of computational examples will be a sequence $\mathcal{W}_{g}$ of hyperelliptic curves called Wiman curves. In this section, we develop the theoretical background on these curves that we will need in these applications. To begin with, we need to have suitable equations for their pluricanonical models, and this part of the story depends only on their being hyperelliptic.

\subsection{Pluricanonical Equations of Hyperelliptic Curves}

Fix a smooth hyperelliptic curve $C$ and a canonical multiple $\nu$, and let $\phi_{\nu}: C \rightarrow \mathbb{P}\left(V_{\nu}\right)=\mathbb{P}^{N_{\nu}}$ be the $\nu$-canonical embedding in $V_{\nu}=H^{0}\left(C, \omega_{C}^{\otimes \nu}\right)^{\vee}$. By Riemann-Roch, $N_{\nu}$ $+1=(2 \nu-1)(g-1)$ and $\phi_{\nu}(C)$ has degree $d=\nu(2 g$ $-2)$. It is easy to write equations for $\phi_{\nu}(C)$, and indeed equations for the embeddings of hyperelliptic curves by more general linear systems. Here we do so in a form convenient for our applications in the next section, following [Stevens 03, pp. 137-138] and [Eisenbud 80]. To simplify notation, we fix $\nu$ and omit it where possible.

Let $\pi: C \rightarrow \mathbb{P}^{1}$ be the $g_{2}^{1}$ on $C$. For convenience, write $k:=\nu(g-1)$ and $e:=g+1$. Then $\phi(C)$ lies on the scroll $S=\mathbb{P}\left(\pi_{*}\left(\omega_{C}^{\otimes \nu}\right)\right)$, where $\pi_{*}\left(\omega_{C}^{\otimes \nu}\right) \cong \mathcal{O}(k) \oplus \mathcal{O}(k+e)$; thus $S \cong \mathbb{P}(\mathcal{O} \oplus \mathcal{O}(-e))$.

Let $C$ be given by the affine equation $y^{2}=f(x)$, where $f(x)$ is polynomial in $x$ of degree $2 g+2$ (or $2 g+1$ if the point at infinity is a branch point). Then a basis of $H^{0}\left(C, \omega^{\otimes \nu} C\right)$ is given by

$$
B_{\nu}:=\left\{1, x, x^{2}, \ldots, x^{k}, y, y x, y x^{2}, \ldots, y x^{k-e}\right\} .
$$

These sections are clearly independent, and we check that

$$
\begin{aligned}
(k+1)+(k-e+1) & =2 \nu(g-1)-(g+1)+2 \\
& =(2 \nu-1)(g-1) \\
& =h^{0}\left(C, \omega_{C}^{\otimes \nu}\right) .
\end{aligned}
$$

Abusing notation, we use these basis elements as variables on $\mathbb{P}^{N}$.

Equations for the scroll are also classical; modern references are [Harris 95, Exercise 9.11] and [Arbarello et al. 85, pp. 96-100]. Suppose $(\nu-1)(g-1)>2$, so that $k>e$. Then the scroll equations are given by the $2 \times 2$ minors of the deleted catalecticant matrix

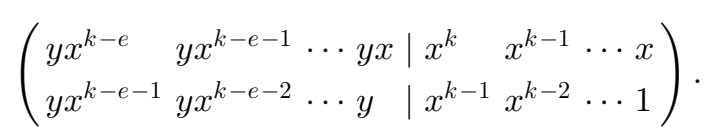


We denote this matrix by $M$ and the ideal generated by its $2 \times 2$ minors by $I_{S}$.

Next, choose a set $Q$ of quadrics encoding the following equations:

$$
\begin{aligned}
y^{2} & =f(x), \\
y^{2} x & =x f(x), \\
& \cdots \\
y^{2} x^{2(k-e)} & =x^{2(k-e)} f(x) .
\end{aligned}
$$

The particular choices of quadrics used to encode these equations won't matter once these are combined with the scroll equations. Write $I_{Q}$ for the ideal generated by these quadrics. The references cited above yield, in particular, the following result:

Lemma 6.1. Suppose the hyperelliptic cover yielding $C$ is branched at $\infty$, so $\operatorname{deg} f(x)=2 g+1$. Then $I\left(\phi_{\nu}(C)\right)=$ $I_{S}+I_{Q}$. That is, the ideal of $\phi_{\nu}(C)$ is given by the scroll equations (the $2 \times 2$ minors of $M$ ) together with the $2(k$ $-e)+1$ additional quadratic equations coming from $Q$.

As an example, in [Morrison and Swinarski 09, CodeSample 6], we find the bicanonical equations of $\mathcal{W}_{4}$, which, as we shall see in (6-4), is the curve given by $y^{2}=x^{9}-1$.

\subsection{Bicanonical Multiplicity-Freeness of Wiman Curves}

We write $\mathcal{W}_{g}$ for the Wiman curve of type $I$ in genus $g$. These curves are named for Anders Wiman, who in 1895, in his first published paper, ${ }^{2}$ [Wiman 95], showed that $\mathcal{W}_{g}$ has the cyclic automorphism group of largest order $4 g+2$ among all smooth curves of genus $g$.

The curve $\mathcal{W}_{g}$ is the smooth hyperelliptic curve given by the affine equation

$$
y^{2}=x^{2 g+1}-1 .
$$

It is often convenient to think of $\mathcal{W}_{g}$ as the hypersurface in the weighted projective space $\mathbb{P}(1, g+1,1)$ given by $y^{2}$ $=x^{2 g+1} z-z^{2 g+2}$, and when we do, we call $[1: 0: 0]$ the branch point at infinity. As we have already remarked, $\operatorname{Aut}\left(\mathcal{W}_{g}\right)$ is cyclic of order $4 g+2$ : fixing a primitive $(4 g+$ 2)nd root of unity $\zeta$ determines a generator $\sigma \in \operatorname{Aut}\left(\mathcal{W}_{g}\right)$ that acts with weight $2 g+1$ on $y$ (that is, as -1 ) and with weight 2 on $x$. The key facts we will need about this action are summarized in the following proposition:

\footnotetext{
${ }^{2}$ His last appeared 59 years later!
}

Proposition 6.2. Let $B_{2}$ be the basis of $V=H^{0}\left(\mathcal{W}_{g}, \omega^{\otimes 2}\right)$ given by (6-1) and let $T$ be the corresponding torus in $\mathrm{SL}(V)$. Then:

1. $\operatorname{Aut}\left(\mathcal{W}_{g}\right)$ fixes the branch point at infinity.

2. The elements of $B_{2}$ are eigenvectors for the action of $\sigma$ on $H^{0}\left(\mathcal{W}_{g}, \omega^{\otimes 2}\right)$ with distinct powers of $\zeta$ as eigenvalues.

Hence, the bicanonical model of $W_{g}$ is multiplicity-free, and the torus $T$ determines stability for it.

Proof: Since $\sigma$ clearly fixes the point at infinity, the description of its action above makes the first statement clear and shows that its action on $H^{0}\left(\mathcal{W}_{g}, \omega^{\otimes 2}\right)$ in the basis $B_{2}$ of $(6-1)$ is by $\zeta^{2 i}$ on $x^{i}$ for $i=0,1, \ldots, k=$ $2 g-2$ and by $\zeta^{2 i+2 g+1}$ on $y x^{i}$ for $i=0,1, \ldots, k-e=g-$ 3 . This gives the second statement, and it, in turn, shows that the elements of $B_{2}$ span invariant lines on each of which $\operatorname{Aut}\left(\mathcal{W}_{g}\right)$ acts by a different character. From this, the final claims follow immediately.

\section{RESULTS AND PREDICTIONS FROM THE LOG MINIMAL MODEL PROGRAM}

In this section, we recall some results from completed stages of the log minimal model program for the moduli stack $\overline{\mathcal{M}}_{g}$ of stable curves and its coarse moduli scheme $\bar{M}_{g}$, and some predictions about conjectural stages. For a more detailed overview of this program, and on the moduli spaces and divisor classes discussed here, see [Hassett and Hyeon 09, Hassett and Hyeon 08] and the recent survey. Because it makes the coefficients involved slightly simpler, we shall state predictions in terms of divisors on the associated moduli stack $\overline{\mathcal{M}}_{g}$.

The goal of the $\log$ minimal model program is to construct the birational models $\overline{\mathcal{M}}_{g}(\alpha)$ determined by the rays $r_{\alpha}$ spanned by the classes $K_{\overline{\mathcal{M}}_{g}}+\alpha \delta$ in the Néron-Severi group of the moduli stack $\overline{\mathcal{M}}_{g}$ of stable curves, where $\delta$ is the divisor class determined by the locus of nodal curves and $1 \geq \alpha \geq 0$. In view of Mumford's formula, $K_{\overline{\mathcal{M}}_{q}}=13 \lambda-2 \delta$, in which $\lambda$ is the Hodge class, the ray $r_{\alpha}$ is also spanned by the class $s_{\alpha} \lambda-\delta$ with slope $s_{\alpha}=\frac{13}{2-\alpha}$. For $\alpha$ near 1 , the ray $r_{\alpha}$ is ample and $\overline{\mathcal{M}}_{g}(\alpha)=\overline{\mathcal{M}}_{g}$, and then, as $\alpha$ decreases, there is a discrete set of critical values of $\alpha$ or $s_{\alpha}$ at which the model $\overline{\mathcal{M}}_{g}(\alpha)$ changes, until at $\alpha=0$ we arrive as the canonical model of $\overline{\mathcal{M}}_{g}$. Understanding the birational alterations involved and constructing the models $\overline{\mathcal{M}}_{g}(\alpha)$ become more delicate as $\alpha$ decreases. 
Predictions about the program can be obtained by assuming a conjectural description of the nef cone of $\overline{\mathcal{M}}_{g}$ called the $F$-conjecture in [Gibney et al. 02] and using consequences of it deduced there (in particular, Theorem 2.2 and Proposition 6.1). These results lead to predictions for the successive smaller critical values $\alpha$, for the loci of stable curves contracted at each such value, and for the nonstable singularities that replace them. Details for $\alpha=\frac{7}{10}$, for example, may be found in [Hassett and Hyeon 09, pp. 20-21]. For small $g$, only larger values of $\alpha$ arise, and the birational alteration involved may be special - for example, loci that are usually of higher codimension may be divisors for small $g$.

On the other hand, a calculation due to Mumford [Mumford 77, Section 5] (see also [Morrison 08, Lemma $21]$ ) determines the slope $s(\nu, m)$ of the polarization with which GIT naturally endows the locus of nodal curves in the $\nu$-canonical Hilbert scheme linearized in degree $m$ and shows that the polarization on the $\nu$-canonical Chow scheme has slope $s(\nu, \infty)$ given by letting $m \rightarrow \infty$. His formula for the polarization is

$$
\left(\frac{m-1}{2 \nu-1}\right)\left(\left(6 \nu^{2} m-2 \nu m-2 \nu+1\right) \lambda-\frac{\nu^{2} m}{2} \delta\right) \text {. }
$$

When one of these GIT slopes equals a critical $s_{\alpha}$, the corresponding GIT quotient is a natural candidate for $\overline{\mathcal{M}}_{g}(\alpha)$. The goal then becomes to see that the stable (and, in some cases, semistable) orbits of this quotient parameterize the expected class of curves.

Table 1 summarizes the first few stages of the program. The $g_{\text {min }}$ column gives the minimal genus below which the general picture indicated must be modified in some way. Since most of these modifications will not be significant in applying our results, we pass over them. A tail is Weierstrass if the point of attachment on it is a Weierstrass point, and is general otherwise.

Interpreting the implications of each row in the table for the GIT problems listed in the last column requires a bit of explanation. Fix a row of the table and consider the closure of the $\nu$-canonical locus in the $\nu$-canonical Hilbert scheme, linearized in degree $m$, for the values given in that row. Then, curves in the sublocus contracted in that row (and in subloci contracted in any higher rows) should have unstable Hilbert points. And ceteris paribus, curves with the replacement singularities in that row (and those in any higher rows, and nodes) but no others should have stable Hilbert points. The italicized proviso in this last statement is that such curves must not exhibit some other destabilizing geometric feature. A careful description of what these features are is necessary in constructing the quotient but not in dealing with the pointwise stability calculations in our examples, so we may, and will, omit giving one.

Each row also comes with an implicit limiting case obtained by sending $m \rightarrow \infty$ in the known rows and by sending $\epsilon \rightarrow 0$ in the predicted rows. In the former case, the limit is the corresponding $\nu$-canonical Chow quotient, and in the latter, it is the $\nu$-canonical Hilbert quotient linearized in the limiting degree $m$. In this limiting GIT problem, we expect both curves in the locus contracted in the fixed row and curves with the replacement singularities in the fixed row to be strictly semistable - again, absent any other destabilizing geometric feature. In particular, in the predicted rows, we expect the limiting value of $m$ to give a linearization lying on a VGIT wall in the sense of [Thaddeus 96, Dolgachev and $\mathrm{Hu} 88$ ] and the contracted and singular curves to change from stable to unstable and from unstable to stable, respectively, as the degree $m$ descends across this value.

Again, we recommend the recent survey [Hyeon 10] for more details, especially concerning the last two rows of Table 1 .

Remark 7.1. We note one additional low-genus result. It is shown in [Hyeon and Lee 10, Proposition 19] that in genus 3 , when $\alpha<\frac{17}{28}$, the locus of hyperelliptic curves (which is a divisor in genus 3 only) is contracted in $\overline{\mathcal{M}}_{g}(\alpha)$. The corresponding slope $s_{\alpha}<\frac{28}{3}$ arises by taking $\nu=2$ and $m=\frac{9}{4}$ in (7-1). Our approach to interpreting stability with respect to a fractional linearization of this type is outlined in Remark 3.2. Thus, here we expect hyperelliptic curves to be $m$-stable for $m>\frac{9}{4}, m$-strictly semistable for $m=\frac{9}{4}$, and $m$-unstable for $m<\frac{9}{4}$.

\section{RESULTS}

In this section, we summarize our computational results. ${ }^{3}$

In Example 8.1, we prove that the bicanonical genus-3 Wiman curve $\mathcal{W}_{3}$ is unstable for $m=2$ and stable for $m \geq 3$ (which matches the predictions in [Hyeon and Lee 07]). In Example 8.2, we prove that the bicanonical genus- 4 Wiman curve $\mathcal{W}_{4}$ is stable for $m \geq 2$, and in Example 8.3 , that the genus-5, $-6,-7$, and -8 Wiman curves are stable for small values of $m$ : both examples confirm predictions of Hassett and Hyeon.

In Example 8.4, we show that a genus-5 curve with an elliptic bridge is unstable for all finite $m$, but Chow

${ }^{3}$ Both the source code files used and the resulting output can be found at http://www.math.uga.edu/ davids/gs/gs.html. 


\begin{tabular}{|c|c|c|c|c|c|c|c|}
\hline Status & $\alpha$ & $s_{\alpha}$ & $g_{\min }$ & Locus Contracted & $\begin{array}{l}\text { Replacement } \\
\text { Singularities }\end{array}$ & Alteration & $\begin{array}{l}\text { GIT Parameters } \\
\text { Giving Alteration }\end{array}$ \\
\hline Known & 1 & 13 & - & - & - & - & $\nu \geq 5, m \gg 0$ \\
\hline Known & $\frac{9}{11}$ & 11 & 3 & elliptic tails & $A_{2}:$ ordinary cusps & contraction & $\nu=3$ or $4, m \gg 0$ \\
\hline Known & $\frac{7}{10}$ & 10 & 4 & elliptic bridges & $A_{3}:$ tacnodes & flip & $\nu=2, m \gg 0$ \\
\hline Predicted & $\frac{2}{3}$ & $\frac{39}{4}$ & 5 & Weierstrass genus 2 tail & $A_{4}$ : rhamphoid cusps & flip & $\nu=2, m=6-\epsilon$ \\
\hline$\underline{\text { Predicted }}$ & $\frac{19}{29}$ & $\frac{29}{3}$ & 5 & general genus 2 tail & $A_{5}:$ oscnodes & contraction & $\nu=2, m=4.5-\epsilon$ \\
\hline
\end{tabular}

TABLE 1. Stages of the log minimal model program.

strictly semistable. We also study genus-5 nodal curves with Weierstrass and general genus-two tails in Examples 8.5 and 8.7. The former is unstable for $m<6$, strictly semistable for $m=6$, and stable for $m=7$; it specializes to a curve with a rhamphoid cusp that we show is also $m=6$ strictly semistable in Example 8.6. The latter is unstable for $m<4.5$ and stable for $m=5,6$, 7; it specializes to a curve with an oscnode. Finally, in Example 8.8 , we study a specific genus- 4 ribbon; this is unstable for all finite $m$, but Chow strictly semistable.

\subsection{Smooth Wiman Curves}

Example 8.1. (The genus-3 Wiman curve.) Genus-3 bicanonical curves are not explicitly covered by Section 6 , since $(\nu-1)(g-1)=2$, or equivalently, $k=e$. But we can stretch the algorithm there to cover this case, too: instead of the curve lying on a scroll given by a deleted catalecticant matrix, in genus 3 , the curve lies on a cone over the rational normal curve given by a catalecticant matrix. To this we can add a quadric encoding $y^{2}=f(x)$, yielding that the ideal for $\mathcal{W}_{3}$ in $K[a, b, c, d, e, f]$ is $(a c$ $-b^{2}, a d-b c, a e-b d, b d-c^{2}, b e-c d, c e-d^{2}, f^{2}-$ $\left.a b+e^{2}\right)$.

In this case, it is not necessary to use Monte Carlo methods. When we compute the full state polytope, gfan [Jensen 08] finds 4615 initial ideals, taking about 1.4 $\mathrm{MB}$ to describe. Interestingly, while $I$ is generated by quadrics, some of the initial ideals have much higher regularity - one of the initial ideals has a generator of degree 19. (The Gotzmann number $m_{P}$ for the Hilbert polynomial $8 t-2$ is 26 .)

We find that $\mathcal{W}_{3}$ is unstable for $m=2$, and stable for $m \geq 3$. For $m=3, \ldots, 12$, we computed $\operatorname{State}_{m}(I)$; for $m=13$ through 27 , we found random sets of six initial ideals whose $m$ th Hilbert points contain the barycenter in their convex hull. By the parabola trick, this implies that $\mathcal{W}_{3}$ is stable for all $m \geq 3$. This corroborates the predictions of Hyeon and Lee mentioned at the end of Section 7.
We also computed the worst 1 -ps when $m=2$. The proximum is

$$
p=\left(\frac{12}{5}, \frac{12}{5}, \frac{12}{5}, \frac{12}{5}, \frac{12}{5}, \frac{10}{5}\right) .
$$

We first computed this using the Maple package Convex [Franz 06]; see [Morrison and Swinarski 09, CodeSample 7]. Later, we learned that version 1.1.2 of Convex had a bug in its proximum function, and checked its answer by verifying the Karush-Kuhn-Tucker conditions. ${ }^{4}$ Then $p-\mathbf{0}_{2}=\left(\frac{1}{15}, \ldots, \frac{1}{15},-\frac{1}{3}\right)$, and therefore the worst $1-\mathrm{ps}$ is one that scales the span of the rational normal curve with equal weights and scales the cone with complementary weight.

Using the MUm function from [Hassett et al. 10] and $w=(10,10,10,10,10,12)$, we get $\operatorname{MUm}(I, w, 2)=-4$ and $\operatorname{MUm}(I, w, 3)=24$. Interpolating using Lemma 5.2, this gives $\mu\left(\left[\mathcal{W}_{3}\right]_{m}, \lambda\right)=4(m-1)(4 m-9)$. Thus, with respect to this $1-\mathrm{ps},[C]$ is $m$-stable, strictly semistable, and unstable for $m$ respectively greater than, equal to, and less than $9 / 4$, confirming the predictions discussed in Remark 7.1.

Finally, we wish to observe that in this example, the state polytope admits a Minkowski-sum decomposition: by [Sturmfels 96, p. 16, Ex. 4], we have $\operatorname{State}_{2}(I)=$ $\operatorname{State}_{2}\left(I_{S}\right)+\operatorname{State}_{2}\left(I_{Q}\right)$. We do not know of a similar decomposition for our other examples, or have reason to expect one.

Example 8.2. (The genus-4 Wiman curve $\mathcal{W}_{4}$.) We computed the ideal of $\mathcal{W}_{4}$ in [Morrison and Swinarski 09, CodeSample 6]. The Hilbert polynomial is $12 t-3$, and its Gotzmann number, using Lemma 2.1, is 63 .

We have not been able to compute the full Gröbner fan of this ideal. To get a sense of how large this might be, we computed state polytopes for related embeddings of lower degree. On a genus-4 hyperelliptic curve,

\footnotetext{
${ }^{4}$ See http://www.math.uga.edu/ davids/gs/wiman3/kkt.pdf .
} 
$K \sim 6 P$, where $P$ is any Weierstrass point, so it is natural to compute state polytopes for linear systems of the form $|K+n P|$.

Table 2 makes it clear that computing the full Gröbner fan for the linear system $|2 K|=|K+6 P|$ is out of reach. (Note: $|K|$ is not an embedding, since $C$ is hyperelliptic. The linear systems $|K+P|$ and $|K+2 P|$ are also not embeddings.)

Next we turned to our Monte Carlo strategy. Using random weights, we were able to establish that $\mathcal{W}_{4}$ is stable for $m$ from 2 to 7 . Next, we examined the output from the $m=2$ calculation more closely. Here 336 random weights were used before stability was established. We chose the 25 initial ideals whose second Hilbert points were closest to the barycenter, and randomly whittled down this set to find a set of 9 initial ideals whose $m$ th Hilbert points establish stability for $4 \leq m \leq 64$ [Morrison and Swinarski 09, CodeSample 4]. By the parabola trick, this implies stability for all $m \geq 2$, and hence Chow stability. It seems plausible that there may be a set (perhaps even many sets) of nine initial ideals that establish stability for all $m \geq 2$.

For $g \geq 4$, Hyeon predicts (private communication) that divisors of slope $\leq 9$ contract the hyperelliptic locus. Solving $\frac{20 m-3}{2 m}=9$ yields $m=\frac{3}{2}$, so the prediction is that this curve should be stable for all $m \geq 2$, which matches our findings. Moreover, we can exhibit a $1-$ ps that flips at $m=\frac{3}{2}$. Let

$$
w=(-2,-2,-2,-2,-2,-2,-2,7,7) .
$$

Then $\operatorname{MUm}(I, w, 2)=108, \quad \operatorname{MUm}(I, w, 3)=648, \quad$ and $\operatorname{MUm}(I, w, 4)=1620$, which gives the polynomial

$$
\mu\left(\left[\mathcal{W}_{4}\right]_{m}, \lambda\right)=108(m-1)(2 m-3) .
$$

By comparing $w$ to the setup used for this example (see [Morrison and Swinarski 09, CodeSample 6]), we see that (in the notation of Lemma 6.1) this $1-$ ps puts all negative weights on multiples of $x$ and all positive weights on multiples of $y$.

Example 8.3. (Higher-genus Wiman curves.) We applied our Monte Carlo methods to the Wiman curves $\mathcal{W}_{g}$ for $g=5,6,7,8$. The ideals of these curves may be obtained using the methods of Section 6. Table 3 shows the number of random weights required to establish stability in different degrees $m$. We have also included the Hilbert polynomial for each example and the corresponding Gotzmann number. Our Macaulay2 code tested stability by adding $4(N+1)$ initial ideals at a time, and we record this, too. Lastly, as $m$ grew large, polymake, run with its default settings, returned error messages in each example; the table includes all our error-free runs, with omitted cells indicating that we encountered a polymake error. Although we are confident that such lacunae could be filled by selecting different options within polymake, or by using a different convex geometry software package, we did not think the game worth the candle.

Table 3 suggests that it is easier to establish stability for larger values of $m$ than for $m=2$. For $g=5$, we also checked some slightly larger values of $m$. Rather than generating new sets of random initial ideals, we checked that the initial ideals used in degree 7 establish stability for degrees 4 through 11. Above degree 11, however, polymake returned error messages once again.

We expect that the whittling procedure used in Example 8.2 could also be applied to prove stability in all degrees for the genus- 5 curve, but we have not made a systematic attempt to do so.

\subsection{Reducible Curves}

Example 8.4. (A genus-5 curve with an elliptic bridge.) An elliptic bridge is a genus-1 subcurve that meets the rest of the curve at two nodes. Hassett and Hyeon show that this is unstable for finite $m$, but Chow strictly semistable. We compute an example illustrating their findings.

We build an example as follows: Let $\mathcal{W}_{2}$ be the Wiman curve of genus 2, $P$ the point at infinity. Let $E$ be the elliptic curve given by the equation $y^{2}=x^{3}-x$ (Kulkarni calls curves like this Wiman curves of type II), and let $Q=[1: 0: 0], R=[0: 0: 1]$. Then $C=\mathcal{W}_{2} \cup_{P=Q}$ $E \cup_{R=P} \mathcal{W}_{2}$. We choose coordinates for the bicanonical embedding of $C$ as follows: We choose variables $a-l$ on $\mathbb{P}^{11}$ and map $P$ to the point $[0: 0: 0: 0: 1: 0: 0: 0: 0$ $: 0: 0: 0]$ and map $Q$ to the point $[0: 0: 0: 0: 0: 0: 0$ $: 1: 0: 0: 0: 0]$. The three irreducible components of $C$

\begin{tabular}{|c|c|}
\hline $\mathcal{W}_{2}$ & 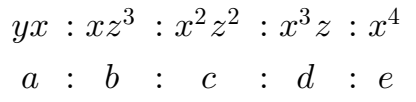 \\
\hline$E$ & 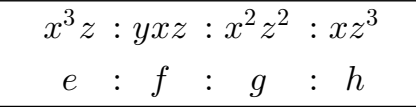 \\
\hline $\mathcal{W}_{2}$ & 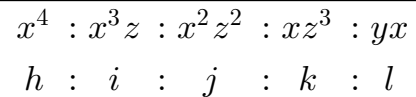 \\
\hline
\end{tabular}
are mapped to $\mathbb{P}^{11}$ as follows:

We know that $\left.\omega_{C}^{2}\right|_{\mathcal{W}_{2}} \cong \omega_{\mathcal{W}_{2}}^{2}(2 P)$, and $\left.\omega_{C}^{2}\right|_{E} \cong$ $\omega_{E}^{2}(2 Q+2 R) \cong \mathcal{O}_{E}(2 Q+2 R)$. Equations for such hyperelliptic curves and linear systems follow from the 


\begin{tabular}{lclc}
\hline Linear System & Number of Initial Ideals & CPU Time & gfan Output File Size \\
\hline$|K+3 P|$ & 3,862 & 3 minutes & $1 \mathrm{MB}$ \\
$|K+4 P|$ & 283,221 & 370 minutes & $101 \mathrm{MB}$ \\
$|K+5 P|$ & $20,694,486$ & 30 days & $25 \mathrm{MB}$ \\
\hline
\end{tabular}

TABLE 2. Growth of complexity of state polytopes of $\mathcal{W}_{4}$.

results in [Stevens 03] and [Eisenbud 80], but we obtained them using MAGMA [Bosma et al. 97, Magma 08]: see [Morrison and Swinarski 09, CodeSamples 5 and 1.1].

Consider the genus-2 subcurve embedded in the span of $h, i, j, k$ and $l$. The point at infinity maps to $[1: 0:$ $0: 0: 0]$. The ideal of $\mathcal{W}_{2}$ in $\mathbb{P}^{4}$ is given by

$$
\left(l^{2}-h i+k^{2}, i^{2}-h j, i j-h k, j^{2}-i k\right),
$$

and the generator of $\operatorname{Aut}\left(\mathcal{W}_{2}\right)$ acts on $h-l$ with weights $8,6,4,2,7$. Then the ideal of $\mathcal{W}_{2}$ in $\mathbb{P}^{11}$ is obtained by adding $(a, b, c, d, e, f, g)$ to this ideal, and the $\operatorname{Aut}\left(\mathcal{W}_{2}\right)$-action is extended to $\operatorname{Span}\{a, b, c, d, e, f, g\}$ by giving these weight 8 .

We can get equations and automorphisms for the other tail by symmetry. The equations and automorphisms of the elliptic-curve component are also easy to find. We obtain

$$
\begin{aligned}
I= & \left(a^{2}-d e+b^{2}, b d-e^{2}, b e-c d, d^{2}-c e, f, g, h, i, j, k, l\right) \\
& \cap\left(g^{2}-e h, f^{2}-e g+g h, a, b, c, d, i, j, k, l\right) \\
& \cap\left(l^{2}-h i+k^{2}, i^{2}-h j, i j-h k, j^{2}-i k, a, b, c, d, e,\right.
\end{aligned}
$$$$
f, g) \text {. }
$$

The following three matrices in GL(12) fix $I$ :

$D_{1}=D\left(\zeta_{10}^{7}, \zeta_{10}^{2}, \zeta_{10}^{4}, \zeta_{10}^{6}, \zeta_{10}^{8}, \zeta_{10}^{8}, \zeta_{10}^{8}, \zeta_{10}^{8}, \zeta_{10}^{8}, \zeta_{10}^{8}, \zeta_{10}^{8}, \zeta_{10}^{8}\right)$, $D_{2}=D\left(\zeta_{10}^{8}, \zeta_{10}^{8}, \zeta_{10}^{8}, \zeta_{10}^{8}, \zeta_{10}^{8}, \zeta_{10}^{8}, \zeta_{10}^{8}, \zeta_{10}^{8}, \zeta_{10}^{6}, \zeta_{10}^{4}, \zeta_{10}^{2}, \zeta_{10}^{7}\right)$, $D_{3}=D\left(-1,-1,-1,-1,-1, \zeta_{4}, 1,-1,-1,-1,-1,-1\right)$.

The representation of the subgroup of automorphisms generated by the three diagonal matrices above is not multiplicity-free. (This can be checked using MAGMA [Magma 08] or GAP [GAP Group 08]; for an example of such a calculation, see [Morrison and Swinarski 09, CodeSample 8].) However, there is a fourth automorphism that flips the two ends of the chain. It is given by the matrix $A$ that swaps $(a, l),(b, k),(c, j),(d, i)$, and $(e, h)$, fixes $g$, and scales $f$ by $\zeta_{4}$.

Let $D_{4}=D_{1} D_{2} D_{3}$, and let $G=\left\langle D_{4}, A\right\rangle$ be the subgroup of GL(12) generated by $D_{4}$ and $A$. Then $G$ is abelian of order 40. However, the basis diagonalizing $G$ is not the basis $a, \ldots, l$ above; thus, in the language of
Definition 4.8 , the torus $T$ scaling the variables $a, \ldots, l$ does not determine stability.

The basis diagonalizing $G$ is $a+l, a-l, b+k, b$ $-k, c+j, c-j, d+i, d-i, e+h, e-h, f, g$. We relabel these as variables $A, B, C, \ldots, L$, write $I^{\prime}$ for the ideal in these coordinates [Morrison and Swinarski 09, CodeSample 1.1], and write $T^{\prime}$ for the torus scaling these variables.

We normalize the matrices $D_{4}$ and $A$ to have determinant 1 , and change them to this basis, yielding:

$$
\begin{aligned}
D_{4}^{\prime}= & D\left(\zeta_{240}^{3}, \zeta_{240}^{3}, \zeta_{240}^{123}, \zeta_{240}^{123}, \zeta_{240}^{171}, \zeta_{240}^{171}, \zeta_{240}^{219}, \zeta_{240}^{219}, \zeta_{240}^{27},\right. \\
& \left.\zeta_{240}^{27}, \zeta_{240}^{207}, \zeta_{240}^{147}\right) \\
A^{\prime}= & D\left(\zeta_{240}^{5}, \zeta_{240}^{125}, \zeta_{240}^{5}, \zeta_{240}^{125}, \zeta_{240}^{5}, \zeta_{240}^{125}, \zeta_{240}^{5}, \zeta_{240}^{125}\right. \\
& \left.\zeta_{240}^{5}, \zeta_{240}^{125}, \zeta_{240}^{65}, \zeta_{240}^{5}\right)
\end{aligned}
$$

The subgroup $G^{\prime}=\left\langle D_{4}^{\prime}, A^{\prime}\right\rangle$ of $\mathrm{SL}(12)$ is multiplicityfree.

The generators of $I^{\prime}$ are far more complicated than the generators of $I$, and the state polytope $\operatorname{State}_{T^{\prime}}\left(I^{\prime}\right)$ is likely to be correspondingly more complicated than $\operatorname{State}_{T}(I)$. Since we found that $I$ already has 500,094 initial ideals, generated in degrees 2 through 9 , we decided to work in its coordinate system as much as possible.

The elliptic bridge is known to be bicanonically unstable in all degrees $m \geq 2$ by [Hassett and Hyeon 08 , Section 10], where the 1-ps with weights $(2,2,2,2,2,1$, $0,2,2,2,2,2)$ is shown to be destabilizing. As a check, we computed $\operatorname{MUm}(I, w, 2)=-12, \operatorname{MUm}(I, w, 2)=-24$, and $\operatorname{MUm}(I, w, 4)=-36$; hence $\mu\left([C]_{m}, \lambda\right)=-12(m-1)$. As a further check, we verified in the extreme degrees, 2 and 9 , that the corresponding state polytope did not contain the barycenter.

The coefficient of $m^{2}$ in $\mu\left([C]_{m}, \lambda\right)$ is 0 . Thus, this $1-\mathrm{ps}$ is not Chow destabilizing. Indeed, this elliptic bridge is Chow strictly semistable, as [Hassett and Hyeon 08, Section 11] shows by deformation-theoretic arguments. To check this, we needed to switch to the torus $T^{\prime}$, since this torus determines stability. We did not attempt to compute the full Chow polytope of $I^{\prime}$, but instead performed a Monte Carlo calculation that found ideals that confirmed strict Chow semistability. 


\begin{tabular}{ccccc}
\hline & $g=5$ & $g=6$ & $g=7$ & $g=8$ \\
\hline$P(t)$ & $16 t-4$ & $20 t-5$ & $24 t-6$ & $28 t-7$ \\
$m_{P}$ & 116 & 185 & 270 & 371 \\
Initial Ideals per Round & 48 & 60 & 72 & 84 \\
& \multicolumn{4}{c}{ Ideals Needed to Establish Stability } \\
$m=2$ & 336 & 480 & 1224 & 1344 \\
$m=3$ & 144 & 300 & 360 & 420 \\
$m=4$ & 48 & 180 & 144 & 168 \\
$m=5$ & 96 & 120 & 144 & 252 \\
$m=6$ & 96 & 60 & 216 & 168 \\
$m=7$ & 48 & 120 & 72 & 168 \\
$m=8$ & 48 & 60 & & \\
$m=9$ & 96 & & & \\
\hline
\end{tabular}

TABLE 3. Number of random weights required to establish stability.

Example 8.5. (A genus-5 curve with a genus-2 tail attached at a Weierstrass point.) Here we consider an example of a nodal genus- 5 curve that has a genus- 3 component and a genus- 2 component (hence a genus-2 tail) where the node is a Weierstrass point of the genus- 2 component. Hassett and Hyeon predict that such a curve is stable for $m>6$, semistable for $m=6$, and unstable for $m<6$ (see Table 1 and [Hyeon 10]). Our calculations confirm this prediction.

We build our $C$ by letting $\mathcal{W}_{3}$ be the Wiman curve of genus 3 with $P$ its the branch point at infinity, letting $\mathcal{W}_{2}$ be the genus-2 Wiman curve with $Q$ its branch point at infinity, and setting $C=\mathcal{W}_{3} \cup_{P=Q} \mathcal{W}_{2}$. The linear series $\omega_{C}^{2}$ is very ample, and the image of $C$ under the corresponding morphism $\phi$ is a degree-16 curve in $\mathbb{P}^{11}$. We know that $\left.\omega_{C}^{2}\right|_{\mathcal{W}_{2}}=\omega_{\mathcal{W}_{2}}^{2}(2 P)$ and $\left.\omega_{C}^{2}\right|_{\mathcal{W}_{2}}=\omega_{\mathcal{W}_{2}}^{2}(2 Q)$. Once again we used MAGMA to obtain the corresponding equations; see [Morrison and Swinarski 09, CodeSample 5].

For $\mathcal{W}_{3}$, coordinatize $\mathbb{P}^{7}$ using the variables $a-h$, and map $\mathcal{W}_{3} \rightarrow \mathbb{P}^{7}$ by sending

$$
y x^{6}, y z x^{5}, z^{5} x^{5}, z^{4} x^{6}, z^{3} x^{7}, z^{2} x^{8}, z x^{9}, x^{10}
$$

to

$$
[a: b: c: d: e: f: g: h]
$$

Then $P$ maps to $[0: 0: 0: 0: 0: 0: 0: 1]$. The ideal of $\mathcal{W}_{3}$ in $\mathbb{P}^{7}$ is given by

$$
\begin{gathered}
\left(-g^{2}+f h,-f g+e h,-f^{2}+e g,-f^{2}+d h,-e f+d g,\right. \\
-e f+c h,-e^{2}+d f,-e^{2}+c g,-d e+c f,-d^{2}+c e,
\end{gathered}
$$

$$
\begin{aligned}
& a g-b h, a f-b g, a e-b f, a d-b e, a c-b d, \\
& \left.b^{2}+c^{2}-f g, a b+c d-g^{2}, a^{2}+d^{2}-g h\right),
\end{aligned}
$$

and the generator of $\operatorname{Aut}\left(\mathcal{W}_{3}\right)$ acts on $a-h$ with weights $5,3,10,12,0,2,4,6$. Then the ideal of $\mathcal{W}_{3}$ in $\mathbb{P}^{11}$ is obtained by adding $(i, j, k, l)$ to the ideal above, and the $\operatorname{Aut}\left(\mathcal{W}_{3}\right)$-action is extended to $\operatorname{Span}\{i, j, k, l\}$ by giving these weight 6 .

Equations and automorphisms of the $\mathcal{W}_{2}$ component can be found in Example 8.4. We intersect the ideals of the two components to get the ideal of $C$ [Morrison and Swinarski 09, CodeSample 1.2]. Its Hilbert polynomial is $P(m)=16 m-4$.

We check that the $\operatorname{Aut}(C)$-action is multiplicity-free. The $\operatorname{Aut}(C)$ representation is generated by the diagonal matrices

$$
D\left(\zeta_{14}^{5}, \zeta_{14}^{3}, \zeta_{14}^{10}, \zeta_{14}^{12}, \zeta_{14}^{0}, \zeta_{14}^{2}, \zeta_{14}^{4}, \zeta_{14}^{6}, \zeta_{14}^{6}, \zeta_{14}^{6}, \zeta_{14}^{6}, \zeta_{14}^{6}\right)
$$

and

$$
D\left(\zeta_{10}^{8}, \zeta_{10}^{8}, \zeta_{10}^{8}, \zeta_{10}^{8}, \zeta_{10}^{8}, \zeta_{10}^{8}, \zeta_{10}^{8}, \zeta_{10}^{8}, \zeta_{10}^{6}, \zeta_{10}^{4}, \zeta_{10}^{2}, \zeta_{10}^{7}\right)
$$

in GL(12). These can be normalized to elements of $\mathrm{SL}(12)$ :

$$
D\left(\zeta_{42}^{16}, \zeta_{42}^{10}, \zeta_{42}^{31}, \zeta_{42}^{37}, \zeta_{42}^{1}, \zeta_{42}^{7}, \zeta_{42}^{13}, \zeta_{42}^{19}, \zeta_{42}^{19}, \zeta_{42}^{19}, \zeta_{42}^{19}, \zeta_{42}^{19}\right)
$$

and

$$
\begin{aligned}
& D\left(\zeta_{120}^{103}, \zeta_{120}^{103}, \zeta_{120}^{103}, \zeta_{120}^{103}, \zeta_{120}^{103}, \zeta_{120}^{103}, \zeta_{120}^{103}, \zeta_{120}^{103}, \zeta_{120}^{79}, \zeta_{120}^{55}\right. \\
& \left.\quad \zeta_{120}^{31}, \zeta_{120}^{91}\right) .
\end{aligned}
$$

The product of these two matrices is diagonal and has distinct eigenvalues; hence, the representation of the cyclic group it generates is multiplicity-free. 
We ran our Monte Carlo program for small values of $m$. We found, as expected, that for $m=6, C$ is strictly semistable, and stable for $m=7$. As a complement, we prove deductively that $C$ is unstable for $m<6$ and strictly semistable for $m=6$. Let $\lambda$ be the $1-$ ps that acts with weights $w=(6,6,6,6,6,6,6,6,4,2,0,5)$. In the notation of [Morrison 08, Proposition 3], $\lambda$ has average weight $\alpha=\frac{59}{12}$ and induces a weight filtration on $\mathcal{O}(m)$ that looks like

$\underbrace{6 m, 6 m, \ldots, 6 m}_{10 m-2}, 6 m-1,6 m-2,6 m-3, \ldots, 6,5,4,2,0$, giving $w(m)=78 m^{2}-15 m-4$. Putting this all together gives

$\mu(C, \lambda)(m)=-(w(m)-m P(m) \alpha)=\frac{2}{3}(m-1)(m-6)$,

with the desired roots and sign for asymptotic stability with a flip at $m=6$.

A check is provided by running the Macaulay2 function MUm on this $C$ and $\lambda$. We find that $\operatorname{MUm}(I, w, 2)=-32$ and $\operatorname{MUm}(I, w, 3)=-48$, which implies by Lemma 5.2 that $\mu([C], \lambda)(m)=8(m-1)(m-6)$. This is 12 times the $\mu$ obtained in the preceding paragraph because MUm shifts the weights by $-\alpha=\frac{59}{12}$ to normalize their sum to be 0 , making it necessary to scale them by 12 to make them integral.

Example 8.6. (A reducible genus-5 curve with a rhamphoid cusp.) The calculation performed for the example above also establishes semistability of a curve with a rhamphoid cusp as well and allows us to confirm the deformation-theoretic analysis of [Hassett and Hyeon 08, Section 10] at the level of ideals.

Let $C$ be the curve of the previous example, and once again let $\lambda$ be the $1-$ ps acting with weights $w=(6,6,6$, $6,6,6,6,6,4,2,0,5)$. Let $C^{\prime}$ be the ideal obtained as the $\lambda$ limit of $C$ (but do not break ties, so that $C^{\prime}$ is not defined by a monomial ideal). The matrices given above for $C$ also fix $I\left(C^{\prime}\right)$, so $C^{\prime}$ is again multiplicity-free. As shown in [Sturmfels 96, Lemma 2.6], $\operatorname{State}_{C^{\prime}}(I)$ appears as a facet of $\operatorname{State}_{C}(I)$. The barycenter is on this facet, so we also get Monte Carlo $m=6$ semistability for $C^{\prime}$.

Finally, we examine $C^{\prime}$ more closely. On all but one of the generators for $I(C)$, the 1 -ps $\lambda$ acts with equal weights on each term, and so these are unchanged in the $\lambda$ specialization. In particular, the $\mathcal{W}_{3}$ component is unchanged in the $\lambda$ specialization. In contrast, the generator $h i-k^{2}-l^{2}$ specializes to $h i-l^{2}$ under $\lambda$. Recalling our embedding of $\mathcal{W}_{2}$, we see that $h i-l^{2}$ encodes $x^{7} z$ $-y^{2} x^{2}$, or $y^{2}=x^{5}$, which is a rhamphoid cusp. Since a rhamphoid cusp adds 2 to the arithmetic genus, and since this component of $C^{\prime}$ is the flat limit of the smooth curve $\mathcal{W}_{2}$ of arithmetic genus 2 , we see that $C^{\prime}$ must be a reducible curve with two irreducible components meeting at a node. One component of $C^{\prime}$ is $\mathcal{W}_{3}$, and the other component is a rational curve with a rhamphoid cusp. Note that the rational component has a $\mathbb{G}_{m}$ of automorphisms fixing the node and cusp that accounts for the $m$ $=6$ strict semistability.

Example 8.7. (A reducible genus-5 curve with a general genus-2 tail.) Here we consider an example of a nodal genus- 5 curve that has a genus- 3 component and a genus2 component (hence a genus-2 tail) where the node is not a Weierstrass point of the genus-2 component. Hassett and Hyeon predict that such a curve is stable for $m>$ 4.5 , semistable for $m=4.5$, and unstable for $m<4.5$. Our calculations again confirm these predictions.

As in Example 8.5, we will use $\mathcal{W}_{3}$ for the genus-3 component, and $P$ its point at infinity. For the genus- 2 component, we use a twisted model of $\mathcal{W}_{2}$. Namely, let $D$ be given by $y^{2}=x^{5}+1$, and let $Q$ be the point $[0: 1: 1]$. The hyperelliptic involution sends $Q$ to $[0:-1: 1]$, and so $Q$ is not a Weierstrass point. On the other hand, $Q$ is fixed under the automorphism $T: D \rightarrow D$ given by $(x$, $y, z) \mapsto\left(\zeta_{5} x, y, z\right)$. We used MAGMA to obtain equations of $D$ under the embedding $|2 K+2 Q|$; see [Morrison and Swinarski 09, CodeSample 5]. We coordinatize $\mathbb{P}^{4}$ using the variables $h-l$, and map $D \rightarrow \mathbb{P}^{4}$ by

$$
\begin{array}{cccccccccc}
y z^{4}+z^{7} & x y z^{3}+x z^{6} & x^{4} z^{3} & x^{3} z^{4} & x^{2} z^{5} \\
h & : & i & : & j & : & k & : & l
\end{array}
$$

Then $Q$ maps to $[1: 0: 0: 0: 0]$. The ideal of $\mathcal{W}_{2}$ in $\mathbb{P}^{4}$ is given by

$$
\left(k^{2}-j l, i^{2}-j k-2 h l, h k-i l, h j-i k\right),
$$

and $T$ acts on $h-l$ with weights $0,1,4,3,2$. Then the ideal of $D$ in $\mathbb{P}^{11}$ is obtained by adding $(a, b, c, d$, $e, f, g)$ to this ideal, and the $T$-action is extended to $\operatorname{Span}\{a, b, c, d, e, f, g\}$ by giving these weight 0 .

We intersect this with the ideal of the genus-3 component to obtain the ideal of $C$ [Morrison and Swinarski 09, CodeSample 1.3]. Its automorphism group is generated by

$$
D\left(\zeta_{42}^{16}, \zeta_{42}^{10}, \zeta_{42}^{31}, \zeta_{42}^{37}, \zeta_{42}^{1}, \zeta_{42}^{7}, \zeta_{42}^{13}, \zeta_{42}^{19}, \zeta_{42}^{19}, \zeta_{42}^{19}, \zeta_{42}^{19}, \zeta_{42}^{19}\right)
$$

and

$$
D\left(1,1,1,1,1,1,1,1, \zeta_{5}^{1}, \zeta_{5}^{4}, \zeta_{5}^{3}, \zeta_{5}^{2}\right)
$$




\begin{tabular}{ll}
\hline$\left\langle b^{2} d^{2}, a d^{2}, a c\right\rangle$ & $\left(m^{2}, 2 m^{2}-m-1,2 m^{2}-4 m+4, m^{2}+2 m-3\right)$ \\
$\left\langle c^{4}, a d^{2}, a c\right\rangle$ & $\left(m^{2}, 3 m^{2}-6 m+5,6 m-8,2 m^{2}-3 m+3\right)$ \\
$\left\langle c^{3}, a c, a^{2} d^{2}\right\rangle$ & $\left(m^{2}+m-2,3 m^{2}-6 m+5,3 m-2,2 m^{2}-m-1\right)$ \\
$\left\langle c^{3}, b^{2} c^{2}, a c, a^{3} d^{2}\right\rangle$ & $\left(m^{2}+3 m-8,3 m^{2}-8 m+11, m+4,2 m^{2}+m-7\right)$ \\
$\left\langle c^{3}, b^{2} c^{2}, b^{4} c, a c, a^{4} d^{2}\right\rangle$ & $\left(m^{2}+6 m-20,3 m^{2}-12 m+27,8,2 m^{2}+3 m-15\right)$ \\
$\left\langle b^{2}, a d^{2}\right\rangle$ & $\left(2 m^{2}-2 m+1,3 m-3,3 m^{2}-6 m+5, m^{2}+2 m-3\right)$ \\
$\left\langle b c d, b^{2}, a b d^{2}, a^{2} d^{3}\right\rangle$ & $\left(2 m^{2}-4, m+2,3 m^{2}-8 m+10, m^{2}+4 m-8\right)$ \\
$\left\langle b c d, b c^{4}, b^{2}, a b d^{2}, a^{2} d^{4}\right\rangle$ & $\left(2 m^{2}+2 m-12,6,3 m^{2}-12 m+26, m^{2}+7 m-20\right)$ \\
$\left\langle c^{3}, b^{2}\right\rangle$ & $\left(3 m^{2}-6 m+5,3 m-3,6 m-7,3 m^{2}-6 m+5\right)$ \\
$\left\langle c^{3}, b^{2} c^{2}, b^{4} c, b^{6}, a c\right\rangle$ & $\left(3 m^{2}-12 m+20,15 m-33,8,3 m^{2}-6 m+5\right)$ \\
$\left\langle c^{6}, b c d, b c^{3}, b^{2}, a b d^{3}\right\rangle$ & $\left(3 m^{2}-7 m+9,6,15 m-37,3 m^{2}-11 m+22\right)$ \\
$\left\langle c^{5}, b c d, b c^{3}, b^{2}\right\rangle$ & $\left(3 m^{2}-6 m+5, m+2,10 m-17,3 m^{2}-8 m+10\right)$ \\
\hline
\end{tabular}

TABLE 4. Initial ideals of a genus-4 ribbon.

The product of these matrices is diagonal with distinct entries, and hence $C$ is multiplicity-free for the cyclic subgroup it generates.

We ran our Monte Carlo program for small values of $m$. We found, as expected, that $C$ is $m$-stable for $m=$ 5,6 , and 7 .

Next, we studied the $1-$ ps $\lambda$ with weights $w=(4,4$, $4,4,4,4,4,4,3,0,1,2$ ) (that is, weights complementary to the weights of $T)$. We computed $\operatorname{MUm}(I, w, 2)=$ $-20, \operatorname{MUm}(I, w, 3)=-24$, and $\operatorname{MUm}(I, w, 4)=-12$; hence $\mu\left([C]_{m}, \lambda\right)=8(m-1)\left(m-\frac{9}{2}\right)$. Thus, this curve is unstable for $m<4.5$.

Once again, we study the $\lambda$ specialization $C^{\prime}$. As in Example 8.5, most generators in the ideal are unchanged in the limit, with the exception of the last generator, $i^{2}-$ $j k-2 h l$, which specializes to $i^{2}-2 h l$ under $\lambda$. Recalling our embedding of $D$, we see that $i^{2}-2 h l$ encodes the oscnode or $A_{5}$ singularity $y^{2}=x^{6}$. We thank Maksym Fedorchuk and Jarod Alper for alerting us to an error in this calculation in an earlier draft of this paper.

\subsection{A Nonreduced Curve}

Example 8.8. (A genus-4 ribbon.) Recall that a ribbon is just a double structure on $\mathbb{P}^{1}$, that is, a scheme $C$ such that $C_{\text {red }} \cong \mathbb{P}^{1}$ and $\mathcal{I}_{C / C_{\text {rad }}}^{2}=0$. Ribbons arise as limits of canonical curves, and thus it is natural to study their GIT stability alongside examples from the log minimal model program.

We study the genus-4 example given in [Bayer and Eisenbud 85, p. 475]. Let $I \subset k[a, b, c, d]$ be the ideal $\left\langle a c-b^{2}, a d^{2}-2 b c d+c^{3}\right\rangle$. This ideal admits a $\mathbb{G}_{m}$-action with weights $-3,-1,1,3$ on the variables $a, b, c, d$, respectively. Its ideal $I$ has twelve initial ideals (listed in Table 4), all generated in degrees $\leq 6$.

For any finite $m$, one can easily check that the ribbon is Hilbert $m$-unstable using the $\lambda$ determined by the $\mathbb{G}_{m}$ above. But we can use the approach described in Remark 5.5 to handle all $m \geq 6$ by computing the vector $\xi_{m}$ associated to each initial ideal in degrees 6 through 8 and interpolating to obtain the polynomial representing $\xi_{m}$ for any $m \geq 6$ (cf. [Kapranov et al. 92, p. 202]). These are also given in the table.

We observe that the polytope that these span, whose dimension we would expect to be 3 , lies in a plane. The "extra" normal vector besides $(1,1,1,1)$ is $(-3,-1,1$, $3)$, the weight vector of the $\mathbb{G}_{m}$-action. Thus, $\operatorname{State}_{m}(I)$ is also only two-dimensional. For example, when $m=6$, we have that $\operatorname{State}_{m}(I)$ is contained in the plane defined by the equations $a+b+c+d=306$ and $-3 a-b+$ $c+3 d=-14$. On the other hand, the barycenter does not satisfy the second equation, and is therefore outside the state polytope.

Finally, we compute the Chow polytope of $I$. Examining the list above, we see that Chow $(I)$ has four vertices, $(1,2,2,1),(1,3,0,2),(3,0,0,3)$, and $(2,0,3,1)$, each approached by one of the four groups of ideals above. This quadrangle contains the barycenter of its ambient plane, so $I$ is Chow semistable. In summary, this ribbon is Hilbert unstable for all finite $m \geq 2$, but Chow strictly semistable. 
Remark 8.9. GIT stability and semistability are open conditions. Thus, in our previous examples, whenever we found that our example was (semi)stable for a given linearization, this implied that a general member of the same component of $\widehat{\mathbb{H}}$ was also (semi)stable. But since the unstable locus is closed, and multiplicity-free examples are very special, the behavior of the particular example above does not indicate that a general ribbon is unstable for finite $m$.

\section{FUTURE STEPS}

We hope to extend this work in several directions. First, we would like to understand other examples with geometry suggested by the log minimal model program. One such class is that of irreducible curves with a rhamphoid cusp and genus at least the $g_{\text {min }}$ of 5 for such curves (cf. Table 1). Another, suggested by [Smyth 08, Smyth 09], is the class of "elliptic triboroughs," curves with a genus-1 component meeting the rest of the curve in three nodes. To date, we can neither find multiplicity-free examples nor show that such examples do not exist, in either class.

Although the examples here provide numerical evidence for conjectural stages of the $\log$ minimal model program, they are far from constructing any of the quotients that would be needed to verify these conjectures. Such constructions remain our ultimate objective.

As a first step, we want to prove the nonemptiness of the stable loci involved by showing that the Wiman curves of all genera are bicanonically stable. To do so, we must better understand the geometry of the initial ideals arising in our examples, with the aim of finding patterns that will allow us to replace our computational proofs of stability by deductive ones. We can identify, in the gf an output for our small-genus examples, initial ideals for which the one-parameter degeneration that produces them can be understood geometrically, in terms that do not depend on $g$. This allows us to write down an analogous degeneration for any $g$, and our goal is to use these to predict the exponent vectors of the corresponding monomial limits. Finally, we will need to be able to construct enough such degenerations to prove that the convex hull of their monomial limits always contains the relevant barycenter. The proof of Chow semistability in Example 8.8 can be viewed as a toy model for this plan.

Second, it will be necessary to pass from the multigraded Hilbert schemes $\widehat{\mathbb{H}}$ used here to the corresponding
Grothendieck Hilbert schemes $\mathbb{H}$, since it is quotients of the latter that naturally carry the polarizations needed to construct further log minimal models. Doing so would require, for example, showing that no codimension-1 component of the complement of the $\ell$-nice locus in $\mathbb{H}$ lies in the $m$-stable locus for the relevant degree $m$. Compared to the previous problem, this is, in some ways, much harder, since it requires dealing with curves exhibiting the menagerie of pathologies typical of the Hilbert scheme, and in others, easier, since what must be checked is that such curves are not stable.

\section{ACKNOWLEDGMENTS}

It is a great pleasure to thank Brendan Hassett, David Hyeon, and Yongnam Lee, whose work got us interested in these GIT questions, and Dave Bayer, Johan de Jong, Bill Graham, Anders Jensen, Julius Ross, Greg Smith, David Smyth, Mike Stillman, and Bernd Sturmfels for helpful discussions. The first author wishes to acknowledge support from a Fordham University Faculty Fellowship during the early stages of this project. The second thanks Sonja Mapes for her expert instruction on programming in Macaulay2, and Dan Grayson for suggesting many improvements to the StatePolytope package. We are grateful to the University of Georgia's Research Computing Cluster for allowing us to use their resources, and to the National Center for Supercomputing Applications for an allocation of computing time under grant TG-DMS090027.

\section{REFERENCES}

[Arbarello et al. 85] Enrico Arbarello, Maurizio Cornalba, Phillip Griffiths, and Joe Harris. Geometry of Algebraic Curves. Vol. I, Grundlehren der Mathematischen Wissenschaften 267, New York: Springer, 1985.

[Baldwin and Swinarski 08] Elizabeth Baldwin and David Swinarski. "A Geometric Invariant Theory Construction of Moduli Spaces of Stable Maps." Int. Math. Res. Pap. IMRP 1 (2008), Art. ID rpn 004, 104. Available at arXiv:0706.1381v3.

[Bayer 82] Davd Bayer. "The Division Algorithm and the Hilbert Scheme." PhD thesis, Harvard University, 1982.

[Bayer and Eisenbud 85] Dave Bayer and David Eisenbud. "Ribbons and Their Canonical Embeddings." Trans. Amer. Math. Soc. 347 (1995), 719-756.

[Bayer and Morrison 88] David Bayer and Ian Morrison. "Standard Bases and Geometric Invariant Theory. I. Initial Ideals and State Polytopes." J. Symbolic Comput. 6 (1988), 209-217.

[Bosma et al. 97] Wieb Bosma, John Cannon, and Catherine Playoust. "The Magma Algebra System. I. The User Language." J. Symbolic Comput. 24 (1997), 235-265. 
[Breuer 00] Thomas Breuer. Characters and Automorphism Groups of Compact Riemann Surfaces, London Mathematical Society Lecture Note Series 280. Cambridge, UK: Cambridge University Press, 2000.

[Dolgachev and $\mathrm{Hu}$ 98] Igor V. Dolgachev and $\mathrm{Yi} \mathrm{Hu}$. "Variation of Geometric Invariant Theory Quotients." Inst. Hautes Études Sci. Publ. Math. 87 (1988), 556 .

[Eisenbud 80] David Eisenbud. "Transcanonical Embeddings of Hyperelliptic Curves." J. Pure Appl. Algebra 19 (1980), $77-83$.

[Fogarty et al. 94] John Fogarty, Frances Kirwan, and David Mumford. Geometric Invariant Theory, Ergebnisse der Mathematik und ihrer Grenzgebiete (2) 34, Berlin: Springer, 1994.

[Gawrilow and Joswig 00] Ewgenij Gawrilow and Michael Joswig. "Polymake: A Framework for Analyzing Convex Polytopes." In Polytopes-Combinatorics and Computation, Oberwolfach 1997, DMV Sem. 29, pp. 43-73. Basel: Birkhäuser, 2000.

[Gibney et al. 02] Angela Gibney, Seán Keel, and Ian Morrison. "Towards the Ample Cone of $\bar{M}_{g, n}$." J. Amer. Math. Soc. 15 (2002), 273-294 (electronic).

[Gieseker 77] David Gieseker. "Global Moduli for Surfaces of General Type." Invent. Math. 43 (1977), 233-282.

[Gieseker 82] David Gieseker. Lectures on Moduli of Curves, Tata Institute of Fundamental Research Lectures on Mathematics and Physics 69. Bombay: Tata Institute of Fundamental Research, 1982.

[Gieseker 83] David Gieseker. "Geometric Invariant Theory and Applications to Moduli Problems." In Invariant Theory, edited by Francesco Gherardelli, Lecture Notes in Mathematics 996, pp. 45-73. New York: Springer, 1983.

[Gotzmann 78] Gerd Gotzmann. "Eine Bedingung für die Flachheit und das Hilbertpolynom eines graduierten Ringes." Math. Z. 158 (1978), 61-70.

[Haiman and Sturmfels 04] Mark Haiman and Bernd Sturmfels. "Multigraded Hilbert Schemes." J. Algebraic Geom. 13 (2004), 735-769.

[Harris 95] Joe Harris. Algebraic Geometry: A First Course, Graduate Texts in Mathematics, 133. New York: Springer, 1995.

[Hassett and Hyeon 08] Brendan Hassett and Donghoon Hyeon. "Log Minimal Model Program for the Moduli Space of Stable Curves: The First Flip." arXiv:0806.3444v1, 2008.
[Hassett and Hyeon 09] Brendan Hassett and Donghoon Hyeon. "Log canonical Models for the Moduli Space of Stable Curves: First Divisorial Contraction". Trans. Amer. Math. Soc. 361 (2009), 4471-4489.

[Hassett et al. 10] Brendan Hassett, Donghoon Hyeon, and Yongnam Lee. "Stability Computation via Gröbner Bases." J. Korean Math. Soc. 47 (2010), 41-62.

[Hyeon 10] Donghoon Hyeon. "An Outline of the Log Minimal Model Program for the Moduli Space of Curves". arXiv:math/1006.1094, 2010.

[Hyeon and Lee 10] Donghoon Hyeon and Yongnam Lee. "Log Minimal Model Program for the Moduli Space of Stable Curves of Genus Three." Math. Res. Lett. 17 (2010), $625-636$.

[Kapranov et al. 92] M. M. Kapranov, B. Sturmfels, and A. V. Zelevinsky. "Chow Polytopes and General Resultants". Duke Math. J. 67 (1992), 189-218.

[Kempf 78] George R. Kempf. "Instability in Invariant Theory." Ann. of Math. (2) 108 (1978), 299-316.

[Maclachlan 65] C. Maclachlan. "Abelian Groups of Automorphisms of Compact Riemann Surfaces." Proc. London Math. Soc. (3) 15 (1965), 699-712.

[Morrison 08] Morrison Ian. "GIT Constructions of Moduli Spaces of Stable Curves and Maps". Surveys in differential geometry. Vol. XIV. Geometry of Riemann surfaces and their moduli spaces, Surv. Differ. Geom., 14, Int. Press, Somerville, MA, 2009, 315-369, 2655332.

[Morrison and Swinarski 09] Ian Morrison and David Swinarski. "Annotated Code Samples to Accompany 'Gröbner Techniques for Low-Degree Hilbert Stability.'," Available online (http://www.math.uga.edu/ davids/ gs/codesamples.pdf).

[Mumford 77] David Mumford. Stability of Projective Varieties, Monographie de l'Enseignement Mathématique 24. Geneva: L'Enseignement Mathématique, 1977.

[Rousseau 78] Guy Rousseau. "Immeubles sphériques et théorie des invariants". C. R. Acad. Sci. Paris Sér. AB 286 (1978), A247-A250.

[Smyth 08] David Ishii Smyth. "Modular Compactifications of $M_{1, n} "$. arXiv:0808.0177, 2008.

[Smyth 09] David Ishii Smyth. "Towards a Classification of Modular Compactifications of the Moduli Space of Curves". arXiv:0902.3690, 2009.

[Stevens 03] Jan Stevens. Deformations of Singularities, Lecture Notes in Mathematics 1811. Berlin: Springer, 2003. 
[Sturmfels 96] Bernd Sturmfels. Gröbner Bases and Convex Polytopes, University Lecture Series 8. Providence: American Mathematical Society, 1996.

[Thaddeus 96] Michael Thaddeus. "Geometric Invariant Theory and Flips". J. Amer. Math. Soc. 9 (1996), 691723 .

[Wiman 95] Anders Wiman. Über die Doppelcurve auf den geradlinigen Flächen. Acta Math. 19 (1895), 63-71.

\section{SOFTWARE PACKAGES REFERENCED}

[Franz 06] Matthias Franz. "Convex: A Maple Package for Convex Geometry". Available at http://www. math.uwo.ca/mfranz/convex. Version 1.1.2, 2006.

[GAP Group 08] The GAP Group. "GAP: Groups, Algorithms, and Programming: A System for Computational Discrete Algebra." Available at http://www.gapsystem.org. Version 4.4.11, 2008.
[Jensen 08] Anders Jensen. "gfan: A Software Package for Computing Gröbner Fans and Tropical Varieties." Available at http://www.math.tuberlin.de/ jensen/software/gfan/gfan.html. Version 0.3, 2008.

[Grayson and Stillman 08] Dan Grayson and Mike Stillman. "Macaulay 2: A Software System for Research in Algebraic Geometry." Available at http://www.math.uiuc.edu/Macaulay2/. Version 1.1, 2008 .

[Magma 08] Computational Algebra Research Group, School of Mathematics and Statistics, University of Sydney. "MAGMA Computational Algebra System." Available at http://magma.maths.usyd.edu.au/magma/. Version $2.15-1,2008$.

[Gawrilow and Joswig 07] Ewgenij Gawrilow and Michael Joswig. "polymake: A Framework for Analyzing Convex Polytopes." Available at http://www.math.tuberlin.de/polymake/. Version 2.3, 2007.

Ian Morrison, Department of Mathematics, Fordham University, Bronx, NY 10458 (morrison@fordham.edu)

David Swinarski, Department of Mathematics, University of Georgia, Athens, GA 30602 (davids@math.uga.edu)

Received January 25, 2010; accepted June 21, 2010. 